\title{
RANS/CAA based Prediction of Jet Mixing Noise in Cruise Flight
}

\author{
Christina Appel*, Karl-Stéphane Rossignol ${ }^{\dagger}$, Alexander Klabes; \\ Andrej Neifeld ${ }^{\S}$, Michaela Herr $₫$, and Roland Ewert" \\ Institute of Aerodynamics and Flow Technology, Technical Acoustics Branch, \\ German Aerospace Center (DLR), Lilienthalplatz 7, 38108 Braunschweig, Germany
}

\begin{abstract}
Besides turbulent boundary layer induced excitation noise, jet noise is the second most important aeroacoustic source for aircraft cabin noise. A goal within the DLR project ECCO (Enhanced Cabin Comfort Computations) was to improve current cabin noise prediction approaches. Within the framework of the project ECCO RANS (Reynolds Averaged Navier Stokes) and CAA (Computational Aero Acoustics) computations for jet noise at cruise conditions have been carried out. Especially at high cruise Mach numbers forward flight effects have a clear impact on the radiated jet noise. These effects are well known from experiments and discussed detailed in the literature. The outcomes of these studies, e.g. scaling laws, will be used to verify the simulated jet noise spectra. Furthermore in-flight surface pressure spectra, which have been recorded during flight tests in the framework of the German national (LuFo IV) project SIMKAB (Simplified Cabin), are discussed here and compared with the simulations. In order to extend the validation data base to a more generic case a single stream jet (SSJ) with different jet and coflow velocities numerically and experimentally investigated.
\end{abstract}

\section{Nomenclature}

$\begin{array}{lll}\xi, \eta, \zeta & {[\mathrm{m}]} & \text { Space separations between two correlated points } \\ \phi & {\left[^{\circ}\right]} & \text { Azimuth angle } \\ \tau & {[\mathrm{s}]} & \text { Time delay } \\ \tau_{s} & {[\mathrm{~s}]} & \text { Time scale } \\ \theta_{e} & {\left[^{\circ}\right]} & \text { Emission angle } \\ \psi & {\left[^{\circ}\right]} & \text { Reception angle } \\ \hat{A} & {\left[\mathrm{~Pa}^{2} / \mathrm{s}^{2}\right]} & \text { Source variance } \\ \mathrm{D} & {[\mathrm{m}]} & \text { Nozzle diameter } \\ \mathrm{Dq} / \mathrm{Dt} & {[\mathrm{Pa} / \mathrm{s}]} & \text { Convective derivative the noise source term } q_{s} \\ f & {[\mathrm{~Hz}]} & \text { Frequency } \\ G & {[-]} & \text { Gaussian filter kernel } \\ G_{m} & {[-]} & \text { Radial-azimuthal part of the filter kernel } \\ k & {[-]} & \text { Velocity exponent } \\ l_{s} & {[\mathrm{~m}]} & \text { Length scale } \\ m & {[-]} & \text { Mode order } \\ \text { OASPL } & {[\mathrm{dB}]} & \text { Over all sound pressure level }\end{array}$

\footnotetext{
${ }^{*}$ Research Scientist, christina.appel@dlr.de.

${ }^{\dagger}$ Research Scientist, Senior AIAA Member, karl-stephane.rossignol@dlr.de.

${ }^{\ddagger}$ Research Scientist, alexander.klabes@dlr.de.

§Research Scientist, AIAA Member, andrej.neifeld@dlr.de.

『Research Scientist, AIAA Member, michaela.herr@dlr.de.

" Research Scientist, Senior AIAA Member, Senior Scientist DLR, roland.ewert@dlr.de
} 


$\begin{array}{lll}p_{t} & {[\mathrm{~Pa}]} & \text { Total pressure } \\ p_{\infty} & {[\mathrm{Pa}]} & \text { Ambient static pressure } \\ q_{s} & {[\mathrm{~Pa}]} & \text { turbulent pressure fluctuations } \\ \hat{q}_{s} & {[\mathrm{~Pa}]} & \text { RMS of } q_{s} \\ \mathrm{R} & {[\mathrm{m}]} & \text { Distance nozzle exit to microphone position } \\ \mathrm{r} & {[-]} & \text { Radial coordinate } \\ \mathrm{SPL} & {[\mathrm{dB}]} & \text { Sound pressure level } \\ S t r & {[-]} & \text { Strouhal number }=f D / u_{j} \\ \mathrm{TKE} & {\left[\mathrm{m}^{2} / \mathrm{s}^{2}\right]} & \text { Turbulent kinetic energy } \\ T_{t} & {[\mathrm{~K}]} & \text { Total temperature } \\ T_{t \infty} & {[\mathrm{K}]} & \text { Ambient total temperature } \\ \mathrm{TBL} & & \text { Turbulent boundary layer } \\ u & {[\mathrm{~m} / \mathrm{s}]} & \text { Velocity in axial direction } \\ u_{j} & {[\mathrm{~m} / \mathrm{s}]} & \text { Jet velocity } \\ u_{\text {cruise }} & {[\mathrm{m} / \mathrm{s}]} & \text { Cruise velocity } \\ \mathcal{U} & {[-]} & \text { Spatio-temporal white noise }\end{array}$

\section{Introduction}

It is known that a jet in forward flight emits lower noise levels than a statically operating jet. A prediction method to quantify the forward flight effect is important for the certification of aircraft regarding flyover noise. Hence since the early 1970s the forward flight effect is a major issue. Viswanathan and Czech ${ }^{1}$ give a detailed review of existing experimental database and highlight their respective qualities and problems. In their work, Viswanathan and Czech show scaling methods, which provide a collapse of the measured jet noise spectra at all angles and over the entire frequency range. This is based on the method introduced by Viswanathan ${ }^{2,3}$ for jets in static environmental conditions. In contrast to the procedure investigated by Plumbee $^{4}$ in the 1976 , it is not restricted to the OASPL.

For the extension on forward flight effects, Viswanathan and Czech determined the velocity scaling exponent by comparing the measured OASPL reduction under several conditions, e.g. different relative velocity ratios $u_{j} /\left(u_{j}-u_{\text {cruise }}\right)$, temperature ratios, jet Mach number and emission angles. The influence of the temperature and Mach number is small, but there is a variation with the emission angle. Secondly Viswanathan and Czech have shown, that the regular Strouhal number based on the jet velocity is the correct non-dimensional frequency for spectra scaling.

The goal of the present study is to apply the RANS/CAA based prediction method of Ewert et. al ${ }^{5,6}$ to a realistic jet nozzle geometry under cruise flight conditions. The scaling procedure devised by Viswanathan and Czech is used to verify the correct reproduction of the forward flight effect. The range of possible ratios between jet and coflow velocity is quite narrow in real flight tests. To expand the data base on further jet and coflow velocties, additional simulations and measurements for a generic single stream jet are conducted. The acoustic measurements have been done at the AWB. A short overview of the test setup is given in section III.

Besides forward flight velocity effects, ambient conditions due to the flight level and thermodynamic conditions related to the jet engine cycle are considered. Temperature effects are covered by the RANS computations. However, the applied acoustic jet noise source model includes only cold jet mixing noise. An extension to hot jet flows is treated in Ewert et. al. ${ }^{7}$ Furthermore installation effects are not yet considered because the applied computational setup provides only an isolated jet.

The employed numerical approach uses turbulence statistics from RANS computations, (i. e. kinetic energy and turbulent dissipation rate), to model acoustic sound sources. The modeled stochastic sources are generated by means of the Random Particle-Mesh Method (RPM). ${ }^{8}$ They closely realize the two-point cross-correlation function used in the jet noise model developed by Tam \& Auriault ${ }^{9}$ ( $\mathrm{T} \& \mathrm{~A}$ ) fine scale noise model to prescribe the fine-scale sound source. The RPM code is coupled with the DLR CAA solver PIANO. Details about the numerical method are presented in section II. 


\section{Computational Approach}

The applied computational approach is a hybrid method for broadband jet noise prediction (Ewert et al. ${ }^{5,6,11}$ ). It includes the following steps: Firstly a RANS computation which provides mean flow data and statistical turbulence quantities is done. Secondly, fluctuating modal sound sources derived from the Tam \& Auriault ${ }^{9}$ cross-correlation model are efficiently obtained from 2-D stochastic realizations using the Random Particle Method ${ }^{8}$ (RPM). Thirdly, a CAA prediction step using the previously computed fluctuating sound sources is conducted. This last step provides the near-field solution for the jet noise radiation, which can be propagated into the far-field by the Ffowcs-Williams and Hawkings (FW-H) method, ${ }^{11}$ to obtain a spectrum that can be compared with far-field noise measurements.

\section{A. RANS computations}

The RANS computations have been performed with the DLR flow solver TAU. It is an unstructured solver, which can also handle structured grids, once converted to an unstructured format. The advantages of structured meshes for aligned flows like boundary layer or jet flows can be kept. The whole computational domain covers a $15^{\circ}$, or $30^{\circ}$ respectively, circumferential segment of the axis-symmetric jet. The dual stream jets are treated with a $15^{\circ}$, the SSJ with $30^{\circ}$ segment. Higher coflow velocities stabilze the RANS compuation and enable a computation on a narrower grid. Symmetry conditions were applied at the boundaries. The mesh consists of a $2 \mathrm{D}$ grid, which was revolved up to the desired angle. To avoid a singularity at the jet axis the mesh extends only down to 0.0125 nozzle diameter (D) in radial direction. The mesh extent is $15 \mathrm{D}$ in radial and 30D in axial direction. Turbulence modeling is done by means of the SST (Shear-Stress-Transport) turbulence model. ${ }^{14}$

The geometry for the dual stream case is resolved with 115.000 grid points per radial-axial plane and one point per degree in azimuthal direction. The meshing was done with the Gridgen/Pointwise mesh generator. The contour of the core and bypass nozzle is taken from the IAE V2527-A5 engine. As Fig. 1 shows, only the rear part of the engine is modeled. The boundary conditions are defined as engine exhaust conditions, where total pressure and temperature ratios can be specified for the core and bypass jet, respectively. The ratios have been calculated by Becker et al. ${ }^{15}$ using an in-house engine cycle model. The cycle model can predict the thermodynamic parameters for each engine component under realistic cruise flight conditions. Therefore only atmospheric conditions, flight Mach number and fan speed have to be specified.

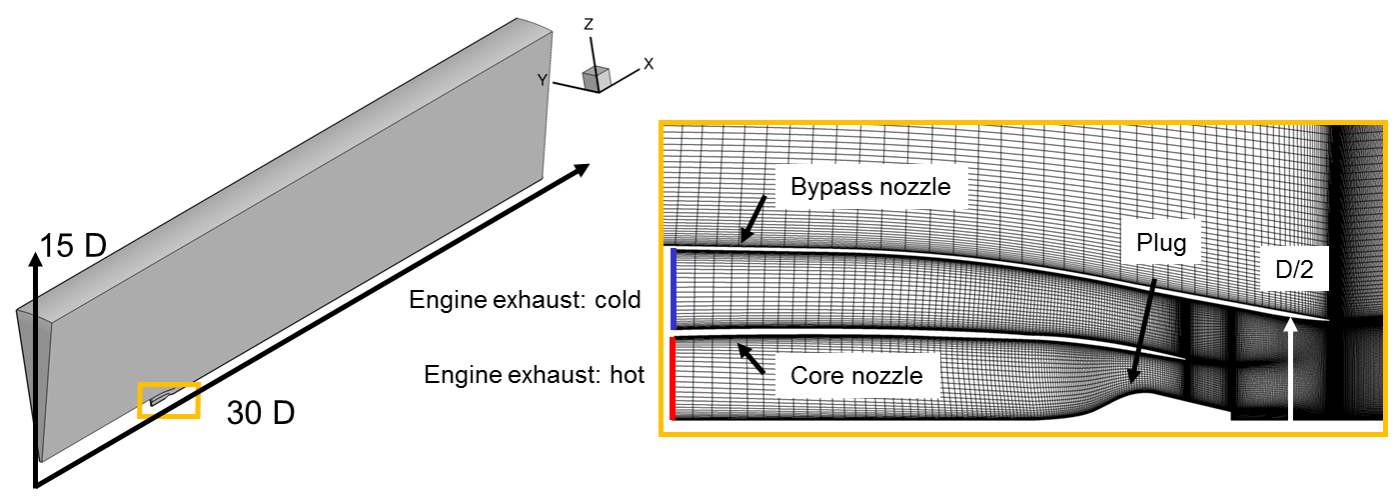

Figure 1. Computational domain for RANS computations.

The simulations with the V2527 engine have been carried out in cruise flight conditions, corresponding to the flight tests. The flight level was $35000 \mathrm{ft}$ (FL350) with standard atmospheric conditions. Five flight Mach numbers were examined: $0.72,0.76,0.78,0.80$ and 0.82 . The related pressure and temperature ratios, as calculated by the cycle model, were specified at the engine exhaust. The reference velocity in the TAU computation is the velocity at the farfield boundary condition, which is defined by the associated flight number. Table 1 summarizes the applied parameters.

For the AWB test case a single stream jet nozzle was used. The nozzle contour based on the experimental setup and the applied mesh is depicted in Fig. 2. The mesh extensions are similar to the dual stream case related to the nozzle diameter. In azimuthal direction the mesh is resolved with one point per degree. The 


\begin{tabular}{|c|c|c|c|c|}
\hline Flight Mach number & $p_{t_{\text {hot }}} / p_{\infty}$ & $T_{t_{\text {hot }}} / T_{t_{\infty}}$ & $p_{t_{\text {cold }}} / p_{\infty}$ & $T_{t_{\text {cold }}} / T_{t_{\infty}}$ \\
\hline \hline 0.72 & 1.966 & 2.55 & 2.11 & 1.1453 \\
0.76 & 2.07 & 2.58 & 2.21 & 1.148 \\
0.78 & 2.13 & 2.598 & 2.27 & 1.1659 \\
0.80 & 2.238 & 2.636 & 2.368 & 1.159 \\
0.82 & 2.38 & 2.7 & 2.494 & 1.17 \\
\hline
\end{tabular}

Table 1. Settings for total pressure and temperature ratios. FL 350 at standard atmosphere conditions.

2D geometry is discretized with 92.000 grid points. The RANS computation neglects the wind-tunnel nozzle. For the computations with coflow, a constant mean flow is imposed.

Under standard atmosphere conditions the jet Mach number is varied in the range between 0.3 and 0.7. The wind tunnel flow is altered between $0 \mathrm{~m} / \mathrm{s}$ (no coflow) and $60 \mathrm{~m} / \mathrm{s}$ for a jet with $\mathrm{Ma}=0.7$.

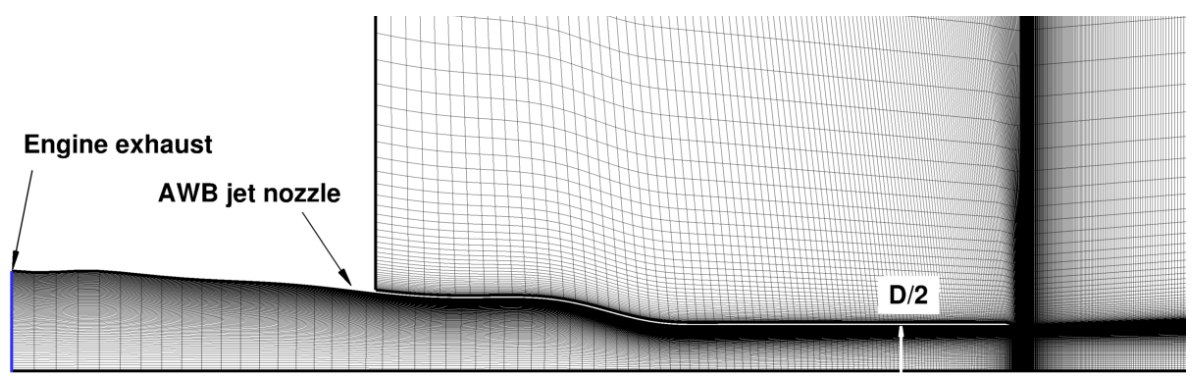

Figure 2. RANS mesh for single stream jet nozzle of the AWB test setup.

\section{B. RANS results}

\section{Dual stream jets in cruise flight}

The axial velocities normalized by the maximum jet velocity are depicted in the left part of Fig. 3 . These figures belong to different cruise flight Mach numbers. From top to bottom, the Mach numbers are 0.72, $0.76,0.78,0.80$ and 0.82 . The pattern in the contour plots indicate that supersonic conditions are already reached at flight Mach number 0.76. The maximum jet Mach number in this case is 1.1.

Due to the supersonic flow regime, a modification of the mean flow input for the CAA computations is necessary to avoid supersonic perturbance propagation. Otherwise, the length of the sound waves will collapse conflicting with the requirement of resolving a wave length with at least seven CAA mesh points. Consequently the speed of sound within the CAA computation is slightly increased by increasing the meanflow pressure. This is done by means of changing the reference pressure value. The mean flow pressure has been increased by the factor 1.6 at most (cruise flight with $\mathrm{Ma}=0.82$ ). The resulting error in sound pressure level is in the order of $4 \mathrm{~dB}$.

The distribution of turbulent kinetic energy is shown on the right part of Fig. 3. Contrary to the velocity plots the changes between the different cases are quite small. However, the spreading rate of the jet decreases with increasing cruise flight velocity.

Radial velocity profiles are depicted in Fig. 4. The velocity profiles for different cruise flight conditions are compared at the axial positions $\mathrm{x} / \mathrm{D}=2.0,4.0,8.0$ and 16.0.

\section{Single stream jet with and without coflow}

Fig. 5 shows the axial velocity distribution and the distribution of the turbulent kinetic energy for the AWB test case without coflow. Only results for the jet Mach number 0.6 case are depicted. The related distributions for the cases with coflow are displayed in Fig 6 . 

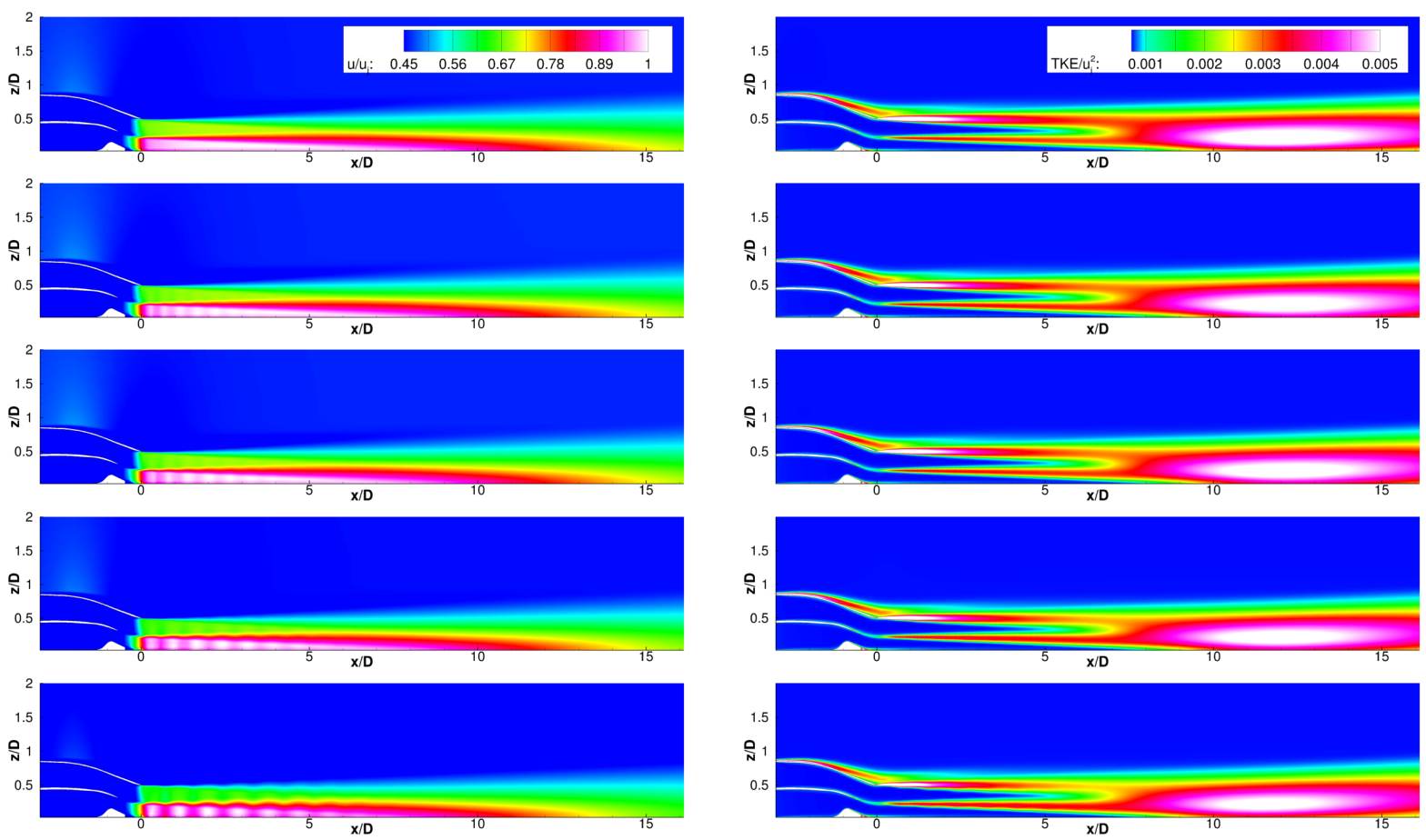

Figure 3. Left: Axial velocity distribution normalized with the maximal jet velocity. Right: Contour plots of the TKE normalized with the squared jet velocity. From top to bottom: flight Mach numbers 0.72, 0.76, 0.78, 0.80 and 0.82 .
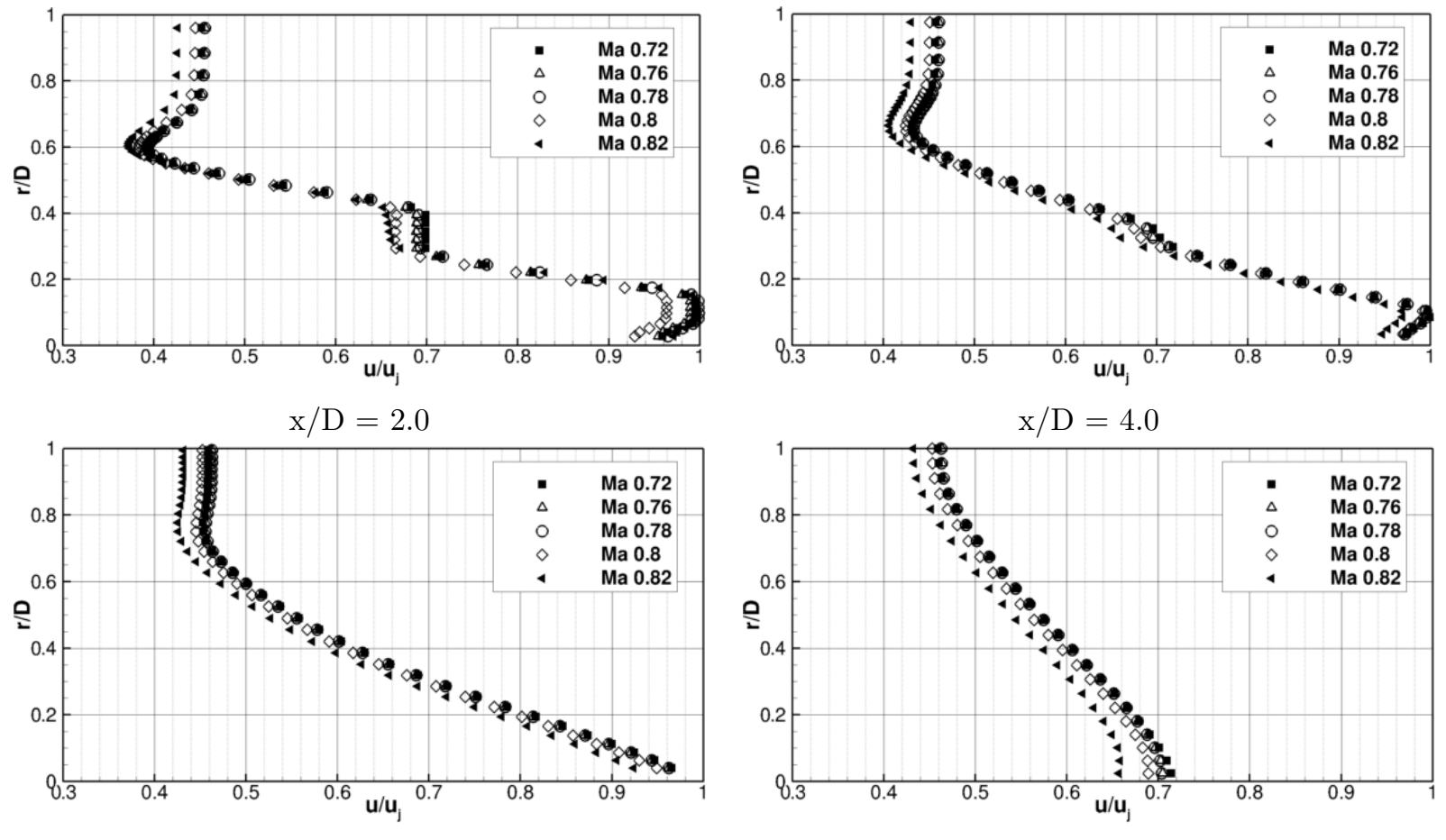

$\mathrm{x} / \mathrm{D}=8.0$

$\mathrm{x} / \mathrm{D}=16.0$

Figure 4. Radial velocity distribution at different flight Mach numbers.

For coflow velocities above $30 \mathrm{~m} / \mathrm{s}$ a clear influence on the velocity and TKE distributions can be seen. The spreading angle is reduced and the potential core length is slightly increased. Furthermore, the TKE 

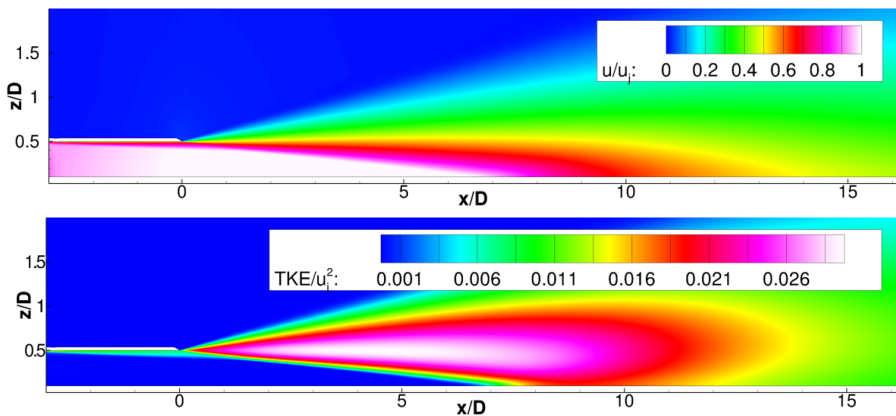

Figure 5. AWB test case at a jet Mach number of 0.6. Top: Axial velocity distribution. Normalized with the maximal jet velocity. Bottom: Contour plot of turbulent kinetic energy (TKE) normalized with the squared jet velocity.
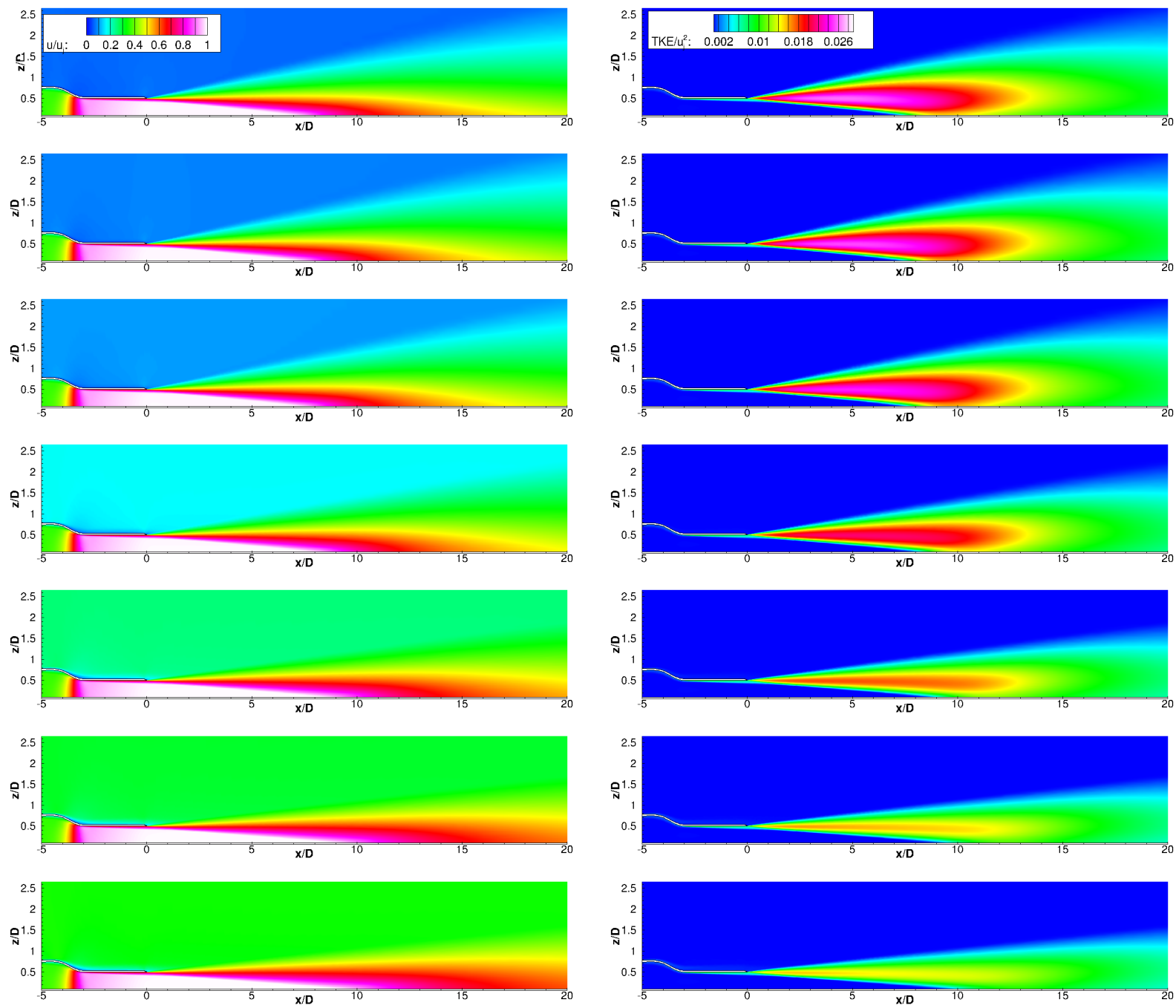

Figure 6. Left: Axial velocity distribution, normalized with the maximal jet velocity. Right: Contour plots of TKE normalized with squared jet velocity. From top to bottom: coflow velocities in $[\mathrm{m} / \mathrm{s}]: \mathbf{1 3 . 5}, 17.0,20.0$, 30.0, 50.0, 60.0, 65.0. Jet Mach number of 0.6.

intensity levels are lowered due to smaller relative velocities in the shear layer. 


\section{CAA computations}

The acoustical source generation is obtained using the RPM method, ${ }^{5,8}$ where the Tam \& Auriault (T\&A) ${ }^{9}$ source model for fine scale jet noise is integrated. The sound propagation is conducted with the CAA solver PIANO. ${ }^{6,11}$

The 3D problem of an axisymmetric jet can be transformed in a set of decoupled (or weakly coupled) axisymmetric problems of discrete mode orders. This is realized by applying Fourier series decomposition in azimuthal direction of the jet flow. Michalke ${ }^{16}$ found from azimuthal decomposition of measured jet noise, that the consideration of a finite number of azimuthal modes, say, the first five modes, is sufficient to represent the observed sound spectra in the far-field. Consequently, just a limited number of azimuthal modes has to be considered in the numerical prediction, thus leading to a significant reduction in computational time.

The genuine form of the T\&A two-point space-time correlation model, which is applied for the generation of the synthetic jet noise sources, is

$$
\left\langle\left(\frac{D q_{s}}{D t}\right)_{1}\left(\frac{D q_{s}}{D t}\right)_{2}\right\rangle=\frac{\hat{q}_{s}^{2}}{c^{2} \tau_{s}^{2}} \times \exp \left\{-\frac{|\xi|}{u_{j} \tau_{s}}-\frac{\ln 2}{l_{s}^{2}}\left[\left(\xi-u_{j} \tau\right)^{2}+\eta^{2}+\zeta^{2}\right]\right\} .
$$

In this equation $\xi, \eta$ and $\zeta$ are the space separations between two correlated points and $\tau$ is the time delay. $l_{s}$ is the length scale, $\tau_{s}$ time scale and the term in front of the exponential function the source variance. These terms are obtained from the RANS solution. The stochastical realization of Eq. (1) in RPM is

$$
\frac{D q_{s}(\boldsymbol{x}, t)}{D t}=\int_{V_{S}} \hat{A} G\left(\boldsymbol{x}-\boldsymbol{x}^{\prime}\right) \mathcal{U}\left(\boldsymbol{x}^{\prime}, t\right) \mathrm{d} \boldsymbol{x}^{\prime} .
$$

Here $\hat{A}$ is the source variance, $G$ a Gaussian filter kernel and $\mathcal{U}$ represents spatio-temporal white noise. ${ }^{17}$ For an azimuthal-modal form of the governing CAA equations, the source term Eq (2) has to be azimuthally decomposed into different Fourier modes $m$. For this, cylindrical coordinates have to be introduced, i.e. $\boldsymbol{x} \rightarrow(x, r, \phi)$. The azimuthal decomposition mainly affects the filter kernel. It can be separated into an axial and radial-azimuthal part, with a Fourier series decomposition of the radial-azimuthal part ${ }^{17}$ :

$$
G=\exp \left(-\frac{\pi}{2} \frac{x^{\prime 2}}{l_{s}^{2}}\right) \cdot G_{m}
$$

where the radial-azimuthal part of the filter kernel is

$$
G_{m}=\sqrt{\frac{r}{2 \pi \cdot l_{s}}} \int_{-\pi}^{+\pi} \exp \left(-\frac{\pi}{2}\left(\frac{r}{l} \cos \phi-\frac{y}{l_{s}}\right)\right) \cdot \exp \left(-\frac{\pi}{2}\left(\frac{r}{l} \sin \phi-\frac{z}{l_{s}}\right)\right) \cdot \exp (-i m \phi) d \phi .
$$

Depending on the chosen mode order $m$ the function $G_{m}$ changes its form, which has an significant influence on the acoustical source distribution. As an example Fig. 7 shows the distribution of the modeled aeroacoustic sources for mode order 0 to 5 . With increasing mode order the length scale and the strength of the sources diminishes. The related filter kernel for specific mode orders are given in Neifeld \& Ewert. ${ }^{17}$ Besides the aeroacoustic source formulation the governing equations for the sound propagation, the linearized Euler equations (LEEs) as well as the FW-H extrapolation into the farfield, have to be transferred into an azimuthal-modal form. Details can be found in Neifeld et al. ${ }^{13,17}$

Though the nozzle geometry is not resolved in the CAA mesh, an existing CAA mesh with the desired resolution can be applied for several geometries. The mesh is normalized with the nozzle diameter, which is in this regard the reference length. This is important for the analysis of the resulting spectra, which have to be dimensioned for comparison with experimental data.

Due to the azimuthal-modal decomposing a $2 \mathrm{D}$ grid for each mode order is necessary. $G_{m}$ is a complex term, so the solutions for real and imaginary part are required. The $2 \mathrm{D}$ meshes for the individual modes can be combined into 3D mesh, where the resulting mesh extends to axial and radial direction plus the mode order as third extension. Real and imaginary part are treated as single blocks. Fig. 8 shows the complete CAA mesh.

The extent of the computational CAA domain is 30D in axial and 5D in radial direction. Moreover the region up to $5 \mathrm{D}$ behind the nozzle exit is resolved to capture the radiation angles between $\theta=60^{\circ} \ldots 120^{\circ}$. The nozzle exit is located at $x / D=0$. This CAA mesh is designed to work with FW-H extrapolation, hence

7 of 22

American Institute of Aeronautics and Astronautics 


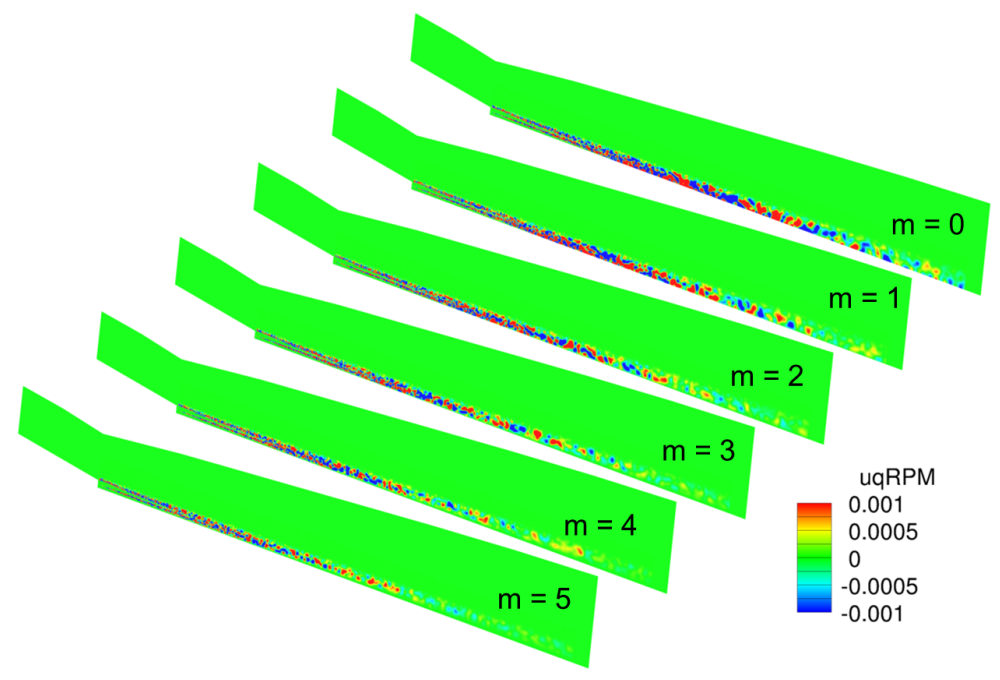

Figure 7. Computed aeroacoustic sources for mode order 0 to 5.

extension in radial direction can be kept small, while numerical resolution can be increased at constant point number. The complete CAA mesh contains 5 Mio. grid points while 10 azimuthal modes were considered. The achieved frequency resolution depends on the reference length, though the CAA mesh is simple scaled to different cases as described before. It is about $2.5 \mathrm{kHz}$ for the dual stream case, but $\approx 35 \mathrm{kHz}$ for the SSJ. This corresponds to a Strouhal number (Str) of Str $\approx 5.7$ for the maximum jet velocity. Sensorpositions acting as virtual microphones have to be specified for the simulation. There fluctuating values like pressure or acoustic particle velocity are recorded during the run.

The computation last about 7 days for parallel run on 60 CPUs. The FW-H extrapolation is done for each individual mode, what can be parallelized by running the required modes at once, respectively one mode per CPU. This took about 2 days. This introduced CAA setup can be used universal depending on the underlaying RANS simulation.

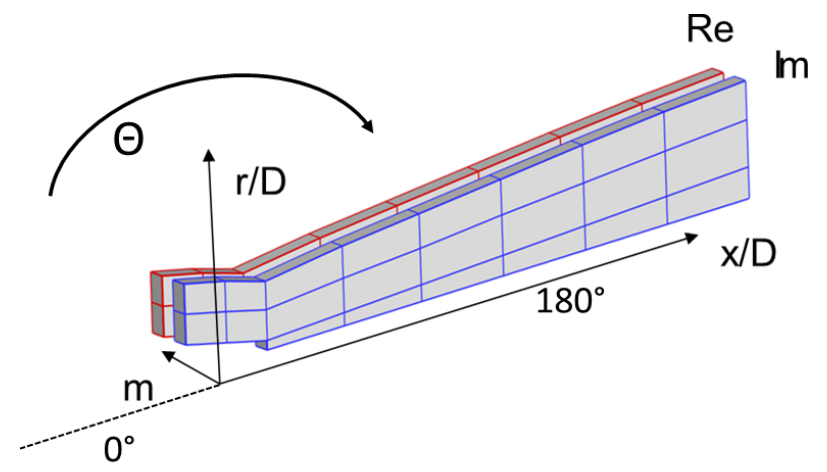

Figure 8. CAA mesh blocks with real and imaginary part.

\section{Experimental setup}

To further validate the CAA computations, experiments with a generic single-stream jet (SSJ) are performed in the AWB. A subsonic cold single jet is simulated by a round straight nozzle $(\mathrm{D}=0.04 \mathrm{~m})$ installed inside the AWB nozzle. The jet nozzle exit plane corresponds with the AWB nozzle exit plane. Fig. 9 shows the shape contour of the SSJ nozzle and for better visualisation a slice of the CAA simulation domain. The 
slice show a snapshot of the non-stationary pressure field of a single computed CAA mode. Using pressurized air, jet Mach numbers of up to 0.7 can be reached. The wind-tunnel free-stream velocity can be varied between 30 and $65 \mathrm{~m} / \mathrm{s}$ to obtain different coflow conditions.

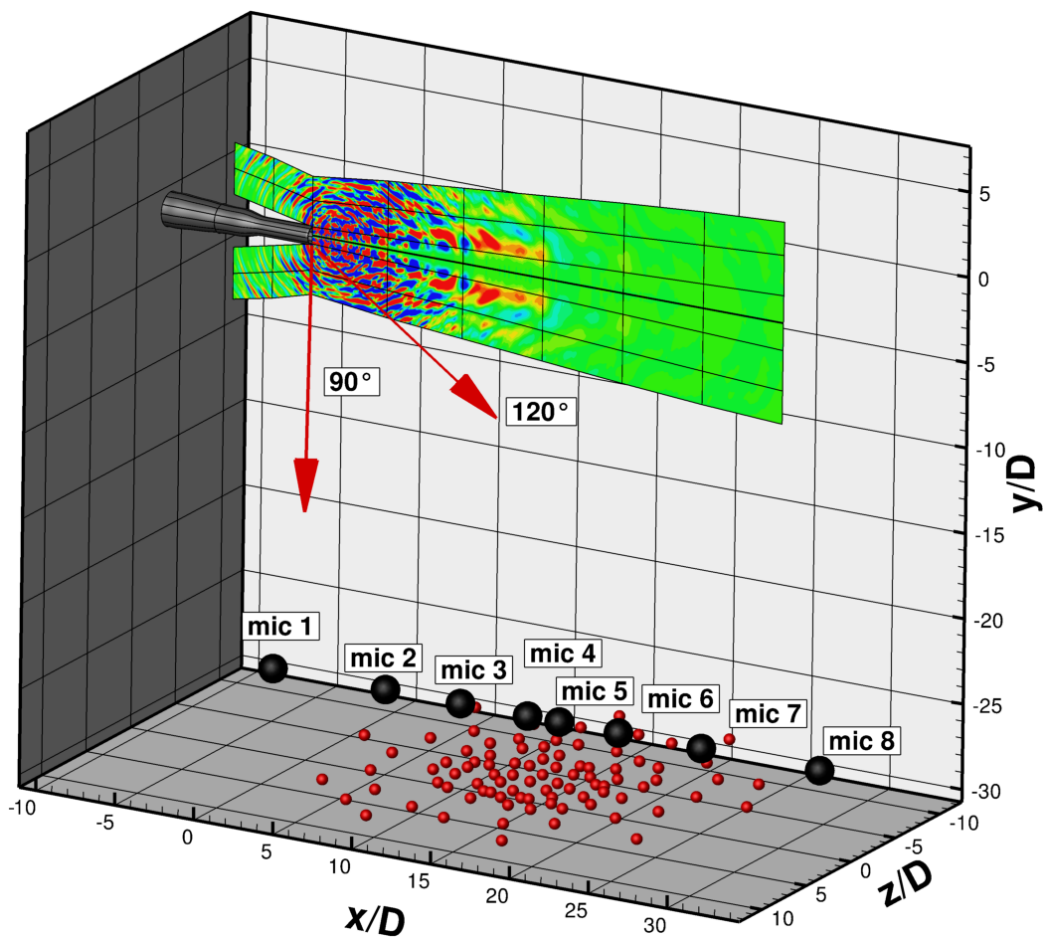

Figure 9. Farfield (black) and array (red) microphones with respect to the SSJ nozzle according to the AWB test setup.

Far-field noise levels are measured using 8 BK Type 4136 1/4" far-field microphones as well as a microphone array with 88 LinearX M51 1/2" microphones. Both the microphone array and the far-field microphones are located below the wind-tunnel test section, aligned with the tunnel center line, as depicted in Fig. 9. Because the far-field noise measurements are contaminated by interference effects, the microphone array data are preferred for comparison with the CAA results. The array data was found to be in excellent agreement with the scaled spectra of Tam and Ariault which provides confidence in the quality of the measurements.

For the cases with coflow, the acoustic data are corrected for wind-tunnel background noise, shearlayer refraction and sound wave convection. For the cases without coflow, no correction is applied to the raw measured data. Processing of the microphone array data is done using the CLEAN-SC ${ }^{12}$ deconvolution algorithm.

\begin{tabular}{|l|c|c|c|c|}
\hline No. & $\mathrm{x} / \mathrm{D}$ & $\mathrm{y} / \mathrm{D}$ & $\mathrm{z} / \mathrm{D}$ & $\mathrm{R} / \mathrm{D}$ \\
\hline 1 & -2.125 & 5 & 25.5 & 25.58 \\
\hline 2 & 5 & 5 & 25.5 & 26.5 \\
\hline 3 & 9.75 & 5 & 25.5 & 27.75 \\
\hline 4 & 14 & 5 & 25.5 & 29.5 \\
\hline 5 & 16 & 5 & 25.5 & 30.5 \\
\hline 6 & 19.75 & 5 & 25.5 & 32.6 \\
\hline 7 & 25 & 5 & 25.5 & 36.0 \\
\hline 8 & 32.5 & 5 & 25.5 & 41.6 \\
\hline
\end{tabular}

Table 2. Postions of the farfield microphones in the AWB test setup. Center of nozzle exit at (0.0/0.0/0.0). 
The second experimental data set was acquired within the project SIMKAB (Simplified Cabin), which was part of aerospace research program (LuFO IV) funded by the Federal Ministry of Economics and Technology (BMWi). A series of flight tests with the DLR research aircraft A320 ATRA were carried out in June 2011 and in October 2012. The main purpose of the flight test was to qualify and quantify the main sources of cabin noise as well as the transfer paths to the passengers under real flight condition. ${ }^{22}$ The flight test setup and the outcomes of the flights from 2011 were published by Spehr et al. ${ }^{22-24}$ and $\mathrm{Hu}$ et al. ${ }^{25}$ In addition to the data described in the aforementioned publications, the flight test carrier ATRA was equipped with flush mounted microphones along the fuselage outer surface. The purpose of these surface microphones was to record the noise caused by the TBL excitation of the fuselage and by the engines. Together with the microphone data acquired inside the cabin and with fuselage structural vibration data, this completes the analysis of the transfer paths.

The scope of the present study is, however, to get jet noise measurements during cruise flight, which can be compared with the simulated jet noise spectra. The surface microphones were Brüel \& Kjær Type 4948. These devices are optimized for sound pressure measurements on surfaces under flight test and wind tunnel conditions. A total of 80 surface microphones were applied on the aircraft fuselage. Some of the microphones were arranged as triplets or groups with four or five units to allow a correlation analysis later on. Fig. 10 shows the measurement positions.

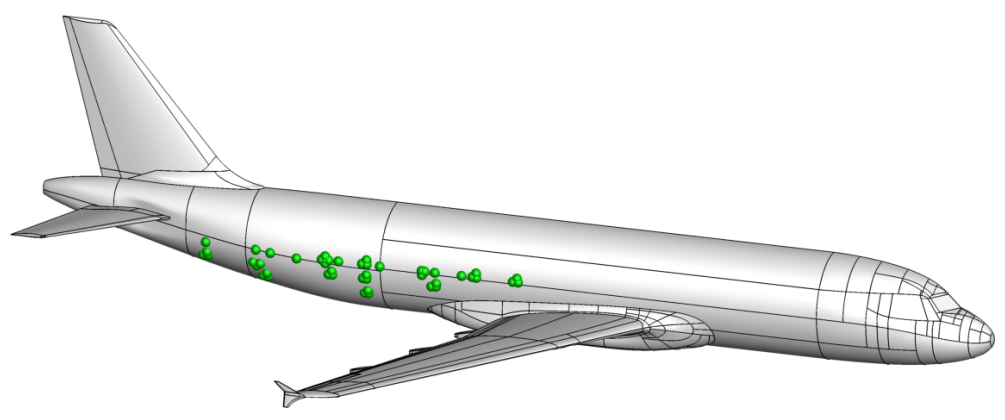

Figure 10. Positions of the microphones on the fuselage surface according to SIMKAB flight test setup.

The ambient conditions for the flight test and the CAA computations are summarized in Table 1. To extract the jet noise spectra, the measured surface pressure data at idle flight conditions was subtracted from the measured surface pressure during cruise flight with constant engine condition. This was done by subtracting the data in terms of power spectral densities. Assuming that the turbulent boundary layer noise stays constant at constant altitude and velocity conditions, the difference corresponds to the jet noise contributions. Though the aircraft descents or decelerates at idle condition for constant speed or constant altitude respectively, the time period were all flight parameters are close to cruise condition is quite short. This leads to a $60 \mathrm{sec}$ time series for the cruise case and $10 \mathrm{sec}$ one for the idle case, resulting in a certain but negligible information loss when averaging. The achieved frequency resolution is $1 \mathrm{~Hz}$.

\section{CAA results}

\section{A. Single stream jet (SSJ) in the AWB}

The SSJ simulations are carried out on the CAA mesh described in section C.The microphones are placed along the CAA domain boundary, depicted in Fig. 11 by the red line. This arrangement of microphones is important, when running a modal FW-H extrapolation. Revolving the microphones $360^{\circ}$ around the bottom line shapes a surface, which encloses all relevant sound sources and provides all required time series e.g. pressure and acoustic particle velocity for an extrapolation into the farfield. A representative snapshot of the pressure fluctuations of the zeroth mode is displayed as a greyscale plot in Fig. 11.

A second set of sensor positions is provided by the farfield microphones from the wind tunnel setup (Fig. 9). The microphone coordinates are given in Tab. 2. These positions are taken as targets for the FW-H extrapolation to allow a comparison of the simulation and experimental data.

As described before the azimuthal-modal approach transforms a 3D problem into several independent 2D problems, which saves a lot of numerical effort. To achieve a representative spectrum at least five mode orders

10 of 22

American Institute of Aeronautics and Astronautics 


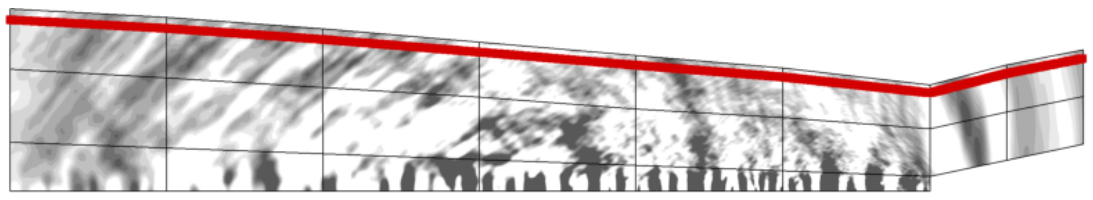

Figure 11. Virtual microphones are placed along a line (red) at the domain boundary. Snapshot of the pressure fluctuations of the zeroth mode.

are necessary. Higher mode orders increase the numerical effort but also increase the frequency resolution. Typical sound pressure fields for the first five modes are exemplary depicted in Fig. 12. The pressure levels decrease with increasing mode order and also the length scales of the structures in the contour plots are getting smaller for higher modes. In the presented CAA simulations 10 modes are considered. To get the spectrum of the 3D axisymmetric jet, the single spectra of each mode order must be summed up. This is illustrated in Fig. 13, where the colored curves mark the spectra of the single modes and the black curve the total spectra.

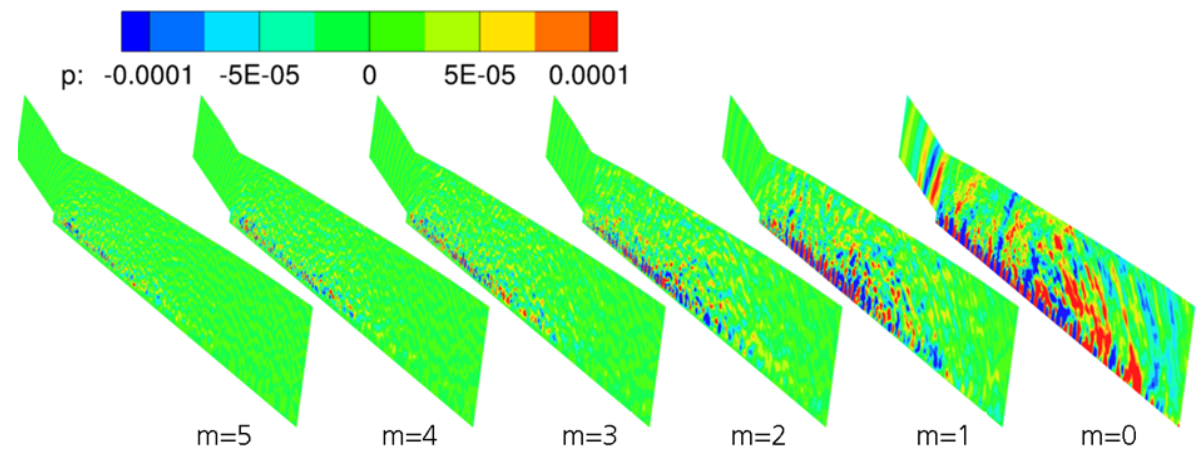

Figure 12. Snapshots of the sound pressure fields for the first five modes.

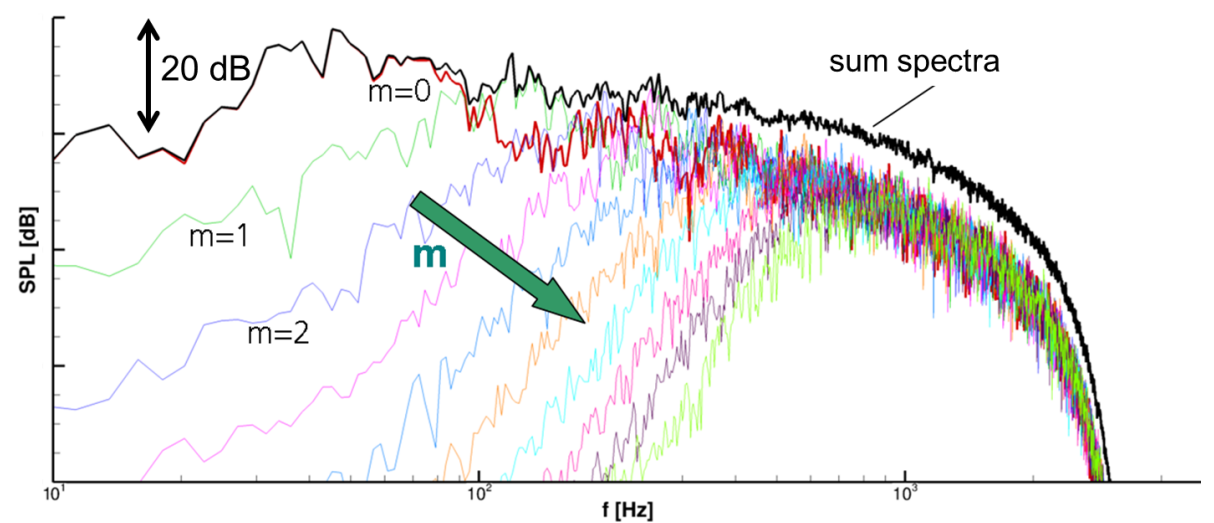

Figure 13. Single modes and summed spectra. Example for a dual stream jet at cruise conditions. Observer position at $\Theta_{e}=90^{\circ}$ measured angle.

Fig. 14 shows the comparison of the sum spectra obtained by the PIANO computations with experimental data. Spectral levels are plotted against a Strouhal number form with the jet velocity $\left(u_{j}\right)$ and the nozzle diameter D.

$$
S t r=\frac{f D}{u_{j}}
$$


To make different data sets comparable a scaling factor (Seiner et al. ${ }^{18}$ ) regarding nozzle diameter and jet velocity is added to the spectral data of the SSJ. Additionally the computed and measured spectra are normalized to a distance of 100 nozzle diameter.

$$
S P L_{\text {norm }}=S P L+10 \cdot \log \left(u_{j} / D\right)
$$

In Fig. 14 the CAA data are compared with the measurements of Seiner et al. ${ }^{18}$ for jet Mach numbers 0.3, 0.5 and 0.7. These cases are without coflow. Additionally fine scale noise spectra from the genuine T\&A model are plotted as black lines. Up to the resolution of the numerical mesh the computation shows good agreement with the model and the experimental data. For a jet Mach number of 0.3 frequencies even up to a Strouhal number of 10.0 are resolved. Deviations occur in the high frequency range $(S t r>2.0)$. However, this high Strouhal number bump possible due to test rig noise is also not part of the genuine T\&A model. Due to the good match of the computed spectra to the T\&A model and the measurements, the Mach number scaling exponent is correctly predicted.

In Fig. 15 the same computed spectra are compared to the AWB measurements for jet Mach numbers $0.3,0.4,0.5,0.6$ and 0.7 . The operating conditions of the used microphones are limited to $16 \mathrm{kHz}$. So, the measured data are truncated to this cut-off frequency. The maxima and the spectral shape are well captured by the simulations.

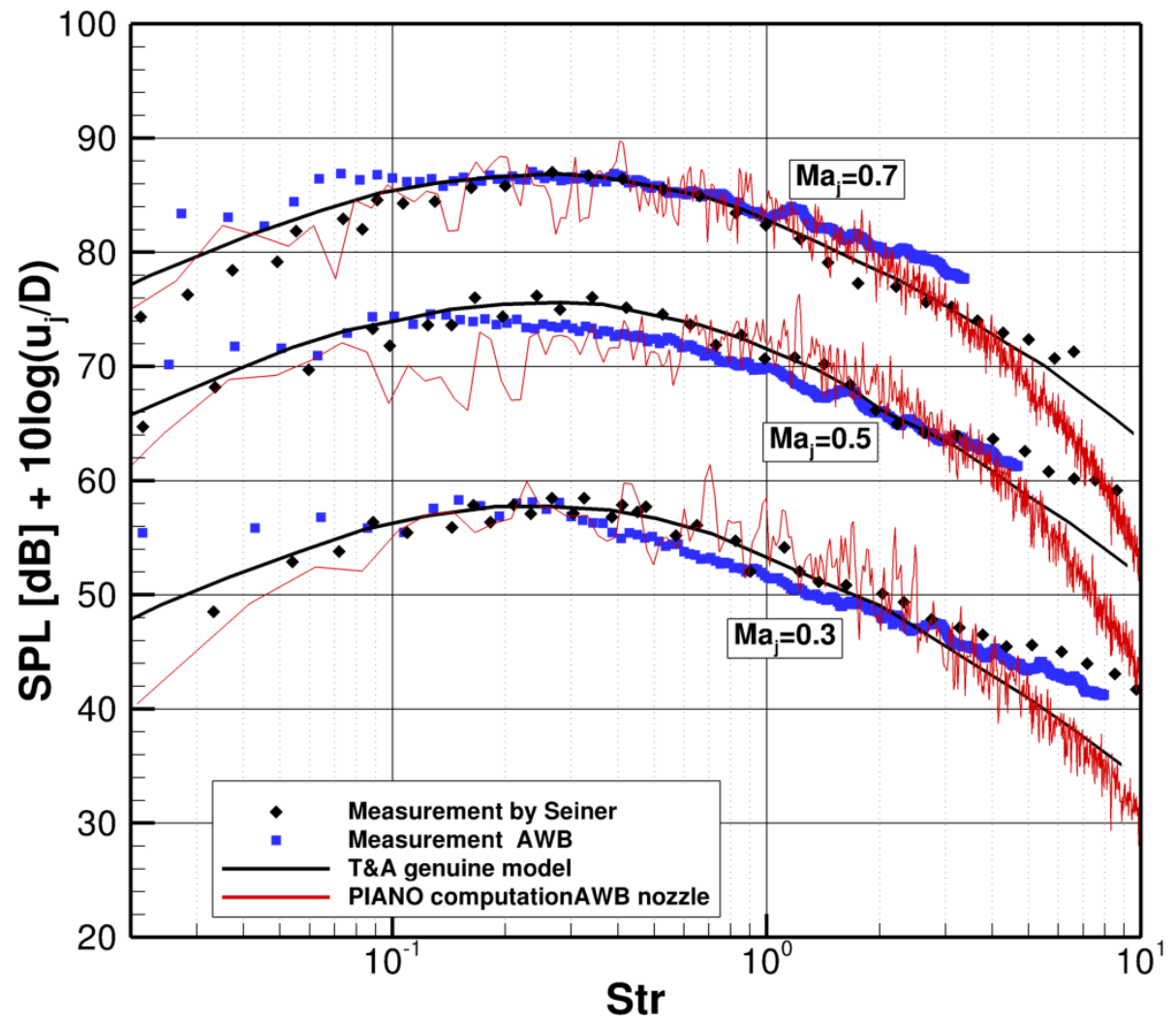

Figure 14. Comparison of SSJ noise spectra without Coflow obtained by PIANO, genuine T\&A model, AWB and Seiner et. al ${ }^{18}$ measurements. Results are normalized to a distance of $100 \mathrm{R} / \mathrm{D}$.

Fig. 16 displays the computed and measured spectra for jet Mach number 0.6 and different wind tunnel velocities. As expected the peak levels drop with increasing coflow velocity. Unfortunately the operational range of the measurement data is reduced for higher coflow velocities due to the wind-tunnel background noise level. However, for the remaining coflow cases the shift of the SPL to lower levels can be captured by the PIANO computation, as the comparisons show a good agreement between simulation and measurement.

For the forward flight effect, a scaling according to Viswanathan \& Czech $^{1}$ is applied. Within this method a velocity exponent $k$ depending mainly on the emission angle is determined. Jet Mach number and temperature ratio have less impact. This exponent scales the ratio of jet and relative velocity Eq. (7). $k$ 


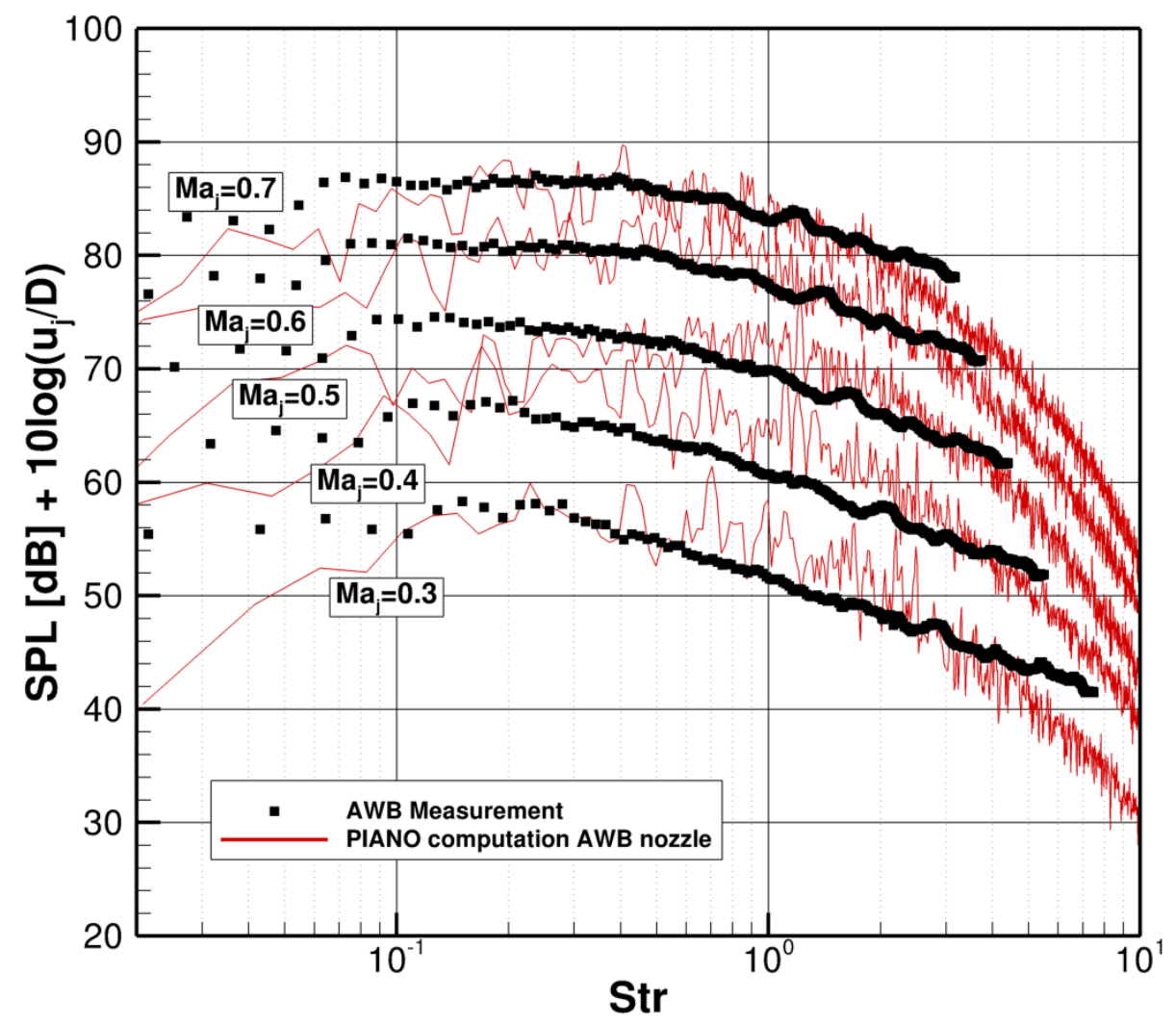

Figure 15. Computed SSJ noise spectra compared with AWB measurements normalized to 100 R/D.

increases slightly from 3.2 up to 3.8 from $60^{\circ}$ to $100^{\circ}$ emission angle. These values for $k$ are taken from Fig. 8 in Viswanathan \& Czech. ${ }^{1}$

$$
\mathrm{SPL}_{\text {scaled }}=\mathrm{SPL}+10 \cdot k \cdot \log 10\left(\frac{u_{j}}{u_{j}-u_{\text {cruise }}}\right)
$$

Fig. 17 shows the measured (a) and computed (b) spectra for different coflow velocities at jet Mach number 0.6 scaled with Eq. (7). In both cases the spectra collapse quite well.

The PIANO/RPM method for fine scale jet noise has been already successfully validated for single and dual stream jets. ${ }^{10,11,17}$ By comparison of computations with and without coflow with experimental data from the AWB measurements and litterature data, it could be demonstrated that the RANS based azimuthal modal CAA prediction method PIANO/RPM is able to reproduce the effect of forward flight on the emitted jet noise.

\section{B. Dual stream jet at cruise conditions}

This part deals with the dual stream jet in cruise conditions corresponding to the SIMKAB flight tests. The selected microphones positions on the ATRA fuselage correspond to the SIMKAB measurement (see Fig. 10). Due to the azimuthal-modal approach the computations can cover an axis-symmetric space. If one revolves the boundary of the CAA mesh introduced in Fig. 8, around the x-axis, the grid dimensions compares with the aircraft dimensions, see Fig. 18. The surface microphone positions are inside the CAA domain. Then an extrapolation with a FW-H method, as it is done in section A, is not necessary, but the $3 \mathrm{D}$ sensor positions have to be transformed into the cylindrical coordinate system.

Due to the high forward flight velocity, the measurement angle $\psi$ is not equal to the emission angle $\theta_{e}$. According to the work of Plumbee ${ }^{4}$ Fig. 19 shows the measured angle $\psi$ at reception time for a jet flow for cruise conditions. Until the signal is received, the source has moved and a new measurement angle $\psi$ results. 
The distance to the observer, who is at rest relative to the nozzle exit changes from $R_{r}$ to $R_{\psi}$. The relation between emission and reception angle can be written as: ${ }^{26}$

$$
\cos \theta_{e}=\cos \psi\left(1-M a_{\text {cruise }}^{2} \sin ^{2} \psi\right)^{1 / 2}-M a_{\text {cruise }} \sin ^{2} \psi
$$

Fig. 20 shows the analysis of Eq. 8 for the investigated cruise flight Mach numbers. Difference from emission to reception angle can be read and equal emission angles for different cruise velocities can be compared.

Jet mixing noise can be described by similarity spectra. ${ }^{18}$ The G-noise spectrum represents the part of the jet noise spectrum caused by fine scale turbulence. This part is radiated under a $60^{\circ}$ to $110^{\circ}$ direction, while the $0^{\circ}$ direction is aligned with the jet axis in upstream direction. $180^{\circ}$ denotes the downstream direction. In Fig. 21 the computed spectrum for the cruise flight case $\mathrm{Ma}=0.78$ is compared at $90^{\circ}$ emission angle with the G-noise similarity spectrum. The forward flight effect is taken into account. Up to the resolved frequency the two spectra match very well.

Fig. 22 depicts the computed narrow band spectra for $\mathrm{Ma}=0.76$ up to $\mathrm{Ma}=0.82$ in the left column. The denoted angles correspond to the emission angles and include the effect of forward flight (s. Fig. 20). Even if the Mach number range of the flight tests is quite narrow, the spectra show clear differences in sound pressure levels, as the left side of Fig. 22 - 26 shows. A scaling according to Eq. (7) is applied in the right side respectivly. Up to an emission angle of $100^{\circ}$, the spectra collapse well, beyond this emission angle the scaling is less successful. Considering the increased angles due to forward flight (Fig.20), the particular microphone position lies far downstream, where the resolution of the CAA mesh might be not sufficient anymore.

Fig. 27 shows the location of the surface pressure sensors at the fuselage. The jet noise content is extracted from the measured data in the manner described in section III. The comparison with the flight test data turns out to be much harder. The compared spectra from the marked sensor positions (Fig. 27) are depicted in Fig. 28. Because virtual and real sensor are in the same frame of reference no further corrections are necessary. Merely $6 \mathrm{~dB}$ are added to the calculated spectra to take the pressure reflection at the solid fuselage surface into account. The spectral shape of both cases is similar, but a gap of about $22 \mathrm{~dB}$ between measurement and simulation remains.

Additional total measured spectra, including jet and TBL induced excitation noise, are shown in Fig. 28 as thin line with diamond shaped symbols. The offset between the total and extracted spectra is about 2 $\mathrm{dB}$ over the whole frequency range. This difference is quite small, but in principle sufficient for the analysis. However, this also implies that jet and TBL induced noise are of the same magnitude. This was also determined by Wilby and Gloyna ${ }^{29}$ in the 1970s. 40 years later the further development of aircraft engines should have led to considerable noise reduction, making it more difficult to extract the jet noise part.

Furthermore, the correlation of the measured signals of adjacent surface pressure sensors appears surprisingly small. Beside the surface pressure sensors, the flight test carrier was also equipped with Kulite sensors flush mounted in three dummy windows. These sensors show a high correlation in streamwise direction for the jet noise and for the TBL induced part. Wilby and Gloyna ${ }^{29}$ describe a method to extract the jet noise from the measured spectra by comparing cross-correlations of cases with strong jet component (e.g. take off or maximal continuous power setting) and without (e.g. idle flight). This method was also applied on the Kulite data. The obtained results are appear reasonable in the time domain, but not in the frequency domain, which raises some doubts about the consistency of some microphone data. Due to confidentiality issues these data sets cannot be shown here.

On the other hand in the work of $\mathrm{Hu}$ et al. ${ }^{25}$ measurements inside the aircraft cabin were conducted. This was also done during the SIMKAB project, so the experimental conditions were the same. In their work they showed by means of correlation analysis, that inside the cabin the extraction of the jet noise part is feasible. The yielded SPL values for the jet noise part at the corresponding measurement positions inside the cabin exhibit the same spectral slope and lie about $15 \mathrm{~dB}$ below the calculated outside pressure levels. Although the complete transfer path of the aircraft cabin is not known yet, this difference appears to be reasonable, thus lending support to the computed noise levels. Due to confidentiality these data sets are not shown here,too.

Regarding the CAA computation the biggest impact might be, that the simulation considers only an isolated engine, instead of the much more complex flight test case. It is expected, that a computation of an isolated jet will not match the absolute sound pressure levels. Although engine operating and coflow conditions are taken from the cruise flight case, the local flow field around the aircraft is neglected. Before reaching the fuselage surface, sound waves have to go through the fuselage boundary layer, whose thickness

14 of 22

American Institute of Aeronautics and Astronautics 
can be estimated to be in the order of $0.3 \mathrm{~m}$, equal to the wavelength of sound waves at a frequency of $1 \mathrm{kHz}$. Siefert et al. ${ }^{27}$ demonstrated that, refraction and scattering effects within the turbulent boundary layer have a clear impact on the received sound waves at the aircraft surface. However the related sensors are placed more or less in downstream direction. Also by taking the retarded source position into account, refraction effects at the boundary layer would yield to lower sound pressure levels at the fuselage surface.

Furthermore the CAA simulation excludes shock cell noise generaly. From RANS computations very slight shock cells for cruise Mach numbers from 0.78 are yielded (Fig. 3). By adapting the reference values supersonic flow will be suppressed within the simulation. The resulting error is in the range of $4 \mathrm{~dB}$. Tam et al. $^{30}$ showed typical spectral features of shock cell noise. These characteristics can not be found in the measured spectra. Due to the engine type IAE V2725-A5 with inner mixing of bypass and core flow, only weak shocks at cruise flight conditions are expected. Additionally the problems with signal processing and extraction might also prevent detecting shock cell noise in the measured data, even if this component would be clearly present.

Another possibly aspect neglected in the present computations are waveguide effects in the turbulent boundary layer. Due to total refraction sound waves also from the second engine of the aircraft can amplify the measured sound pressure fluctuations. Finally installation effects of the engine interacting the pylon, wing and fuselage may play an important role.

In the framework of the DLR project ECCO also jet noise predictions with a complete different semiempirical method, which was introduced by Bassetti and Guérin, ${ }^{31}$ were carried out. They showed encouraging, similar absolute values for the calculated spectra of the isolated ATRA engine at cruise conditions.

\section{Summary and Outlook}

In this study the effects of forward flight for cruise conditions on fine scale jet noise spectra have been investigated. In the framework of the DLR project ECCO CFD and CAA computations for a realistic dual stream jet at cruise condition have been carried out. The applied RANS based prediction method covers the $3 \mathrm{D}$ case with an efficient azimuthal-modal approach. The complex case is split into individual 2D problems, which can be solved for the first few mode orders. This method is already validated for single and dual stream jets with generic nozzle design as well as for complex mixer and nozzle treatments. Within the current study it could be demonstrated, that the resulting jet noise spectra show a similar spectral shape and follow scaling laws, which are provided by several experimental studies. However the prediction fails for the absolute levels measured in the flight test.

Although the simulation considers only an isolated jet, what neglects mainly installation and refraction effects, the extraction of the jet noise content from the measured data turns out harder than might be expected. For the surface pressure sensors this was done by subtracting the data in terms of power spectral densities. However cross-correlation analysis showed that the yield spectra are not reliable. Additional, Kulite data was processed according to the method provided by Wilby \& Gloyna. ${ }^{29}$ Also the procedure does not lead to a clear source ranking. The comparison with measured data from interior microphones provided by $\mathrm{Hu}$ et al. ${ }^{25}$ shows a difference of about $15 \mathrm{~dB}$ between interior and exterior position. Even if the transfer path can not be described complete, this gap appears to be feasible.

To verify the CAA data a generic test case with a SSJ has been set up and wind tunnel tests with homogeneous coflow conditions have been conducted. To crosscheck the AWB jet noise measurements, fine scale noise spectra without coflow are compared with established experimental data from literature. The measured jet noise spectra with and without coflow are compared with simulation data and showed a good agreement.

The ongoing tasks might be to extend the CAA simulation domain to cover the important boundary layer refraction effects. Due to the comparatively near location of the engine relative to the fuselage, one may consider a full 3D simulation, which covers the jet flow and fuselage boundary layer flow. The azimuthal modal approach is, however not applicable for this purpose. Recent research activities about full 3D computations for jet flap interaction noise with stochastic source modeling are discussed in Neifeld et al., ${ }^{28}$ where the scope of application of PIANO/(F)RPM method is extended. Further on an improvement of the experimental data processing is recommended, to extract the jet noise content clearly. 


\section{References}

${ }^{1}$ Viswanathan, K., Czech, M. J., Measurement and Modeling of Effect of Forward Flight on Jet Noise, AIAA 2010-3921

${ }^{2}$ Viswanathan, K., Scaling laws and a Method for Identifying Components of Jet Noise, AIAA Journal, Vol. 44, No. 10, 2006

${ }^{3}$ Viswanathan, K., Improved Method for Prediction of Noise from Single Jets, AIAA Journal, Vol. 44, No. 1, 2007

${ }^{4}$ Plumbee, H. E., Jr., Effects of forward velocity on turbulent jet mixing noise, NASA CR-2702, 1976

${ }^{5}$ Ewert, R., Neifeld, A. and Fritzsch, A., A 3-D modal stochastic jet noise source model, AIAA 2011-2887, 17th AIAA/CEAS Conference.

${ }^{6}$ Neifeld, A. and Ewert, R., Jet Mixing Noise from Single Stream Jets using Stochastic Source Modeling, AIAA 2011-2700, 17th AIAA/CEAS Conference.

${ }^{7}$ Ewert, R., Neifeld, A. and Wohlbrandt, A., A three-parameter Langevin model for hot jet mixing noise prediction, AIAA $2012-2238$

${ }^{8}$ Ewert, R., RPM - the fast Random Particle-Mesh method to realize unsteady turbulent sound sources and velocity fields for CAA applications, AIAA 2007-3506.

${ }^{9}$ Tam, C.K.W. and Auriault, L., Jet mixing noise from fine-scale turbulence, AIAA J. Vol. 37, No. 2, February 1999.

${ }^{10}$ Neifeld, A., Ewert, R., Steger, M., Rose, M.,Prediction of Hot Jet Mixing Noise Using Extended Stochastic Source Correlations, AIAA 2013-2040.

${ }^{11}$ Neifeld, A. and Ewert, R., On the Contribution of Higher Azimuthal Modes to the Near- and Far-Field of Jet Mixing Noise, AIAA 2012-2114, 18th AIAA/CEAS Conference.

${ }^{12}$ Sijtsma, P., CLEAN Based on Spatial Source Coherence, AIAA-2007-3436, 13th AIAA/CEAS Aeroacoustics Conference.

${ }^{13}$ Neifeld, A. and Appel, C., Numerical Jet Noise Predictions with Emphasis on Passive Noise Reduction Applications, ODAS 2013, 13th ONERA-DLR Aerospace Symposium.

${ }^{14}$ Menter, F. R., Two-equation eddy-viscosity turbulence models for engineering applications, AIAA Journal, Vol. 32, No. 8 (1994).

${ }^{15}$ Becker, R.-G., Wolters, F., Nauroz, M., Otten, T., Development of a gas turbine performance code and its application to preliminary engine design, DLRK 2011, 27.-29. September, Bremen, Germany, 2011

${ }^{16}$ Michalke, A., Some Remarks on Source Coherence Affecting Jet Noise, Journal of Sound and Vibration,Vol. 87(1), 1983.

${ }^{17}$ Neifeld, A., Ewert, R., Azimuthal-modal approach for jet mixing noise using hybrid RANS/CAA method in Aeroacoustics research in Europe: The CEAS-ASC report on 2011 highlights', Journal of Sound and Vibration, 331 (21), pp. 4609-4628, 2012.

${ }^{18}$ Tam, C.K.W., Golebiowski, M. and Seiner, J. M., On the Two Components of Turbulent Mixing Noise from Supersonic Jets, AIAA 1996-1716

${ }^{19}$ Pott-Pollenske, M., Friedel, H., Aeroacoustic Characteristics of Externally Blown Flap Systems, AIAA 2012-2729

${ }^{20}$ Amiet, R.K., Correction of Open Jet Wind Tunnel Measurements for Shear Layer Refraction, AIAA Paper No. 75-532, Hampton, VA./USA, March 24-26, 1975.

${ }^{21}$ Ruijgrok, G.J.J., Elements of Aviation Acoustics, Delft University Press, Delft, 1993

${ }^{22}$ Spehr, C., Hennings, H., Buchholz, H., Haxter, S., Hebler, A., In-flight Sound Measurements: A First Overview, AIAA 2208-2012

${ }^{23}$ Spehr, C.,Haxter, S., Two-Dimensional Evaluation of Turbulent Boundary Layer Pressure Fluctuations at Cruise Flight Conditions, AIAA 2012-2139

${ }^{24}$ Spehr, C.,Haxter, S., Examination of the Influence of Flight Altitude and Speed on the Efimtsov Model Parameters, AIAA 2013-2028

${ }^{25} \mathrm{Hu}, \mathrm{N} .$, Buchholz, H., Herr, M., Spehr, C.,Haxter, S., Contributions of Different Aeroacoustic Sources to Aircraft Cabin Noise, AIAA 2013-2030

${ }^{26}$ Ribner, H. S., The Generation of Sound in Turbulent Jets, Advances in Applied Mechanics, Vol. 8, 1694

${ }^{27}$ Siefert, M., Delfs, J., Caruelle, B., Refraction and Scattering in High Mach Number Boundary Layers, AIAA 2011-2847

${ }^{28}$ Neifeld, A., Ewert, R., Keller, D., Steger, M., Towards Prediction of Jet Noise Installation Effect using Stochastoc Source Modeling, (submitted to the 20th AIAA/CEAS Conference)

${ }^{29}$ Wilby, J. F., Gloyna, F. L., Viobration measurents of an airplane fuselage structure II. Jet noise excitation, Journal of Sound and Vibration (1972) 23 (4), 467-486.

${ }^{30}$ Tam, C., Pastouchenko, N., Viswanathan, K., Broadband shock-cell noise from dual stream jets, Journal o fSound and Vibration 324(2009)861891.

${ }^{31}$ Bassetti, A., Guérin, S., Semi Empirical Jet Noise Modelling for Cabin Noise Prediction - Acoustic Loads in the Geometric Near Field, AIAA 2011-2925 

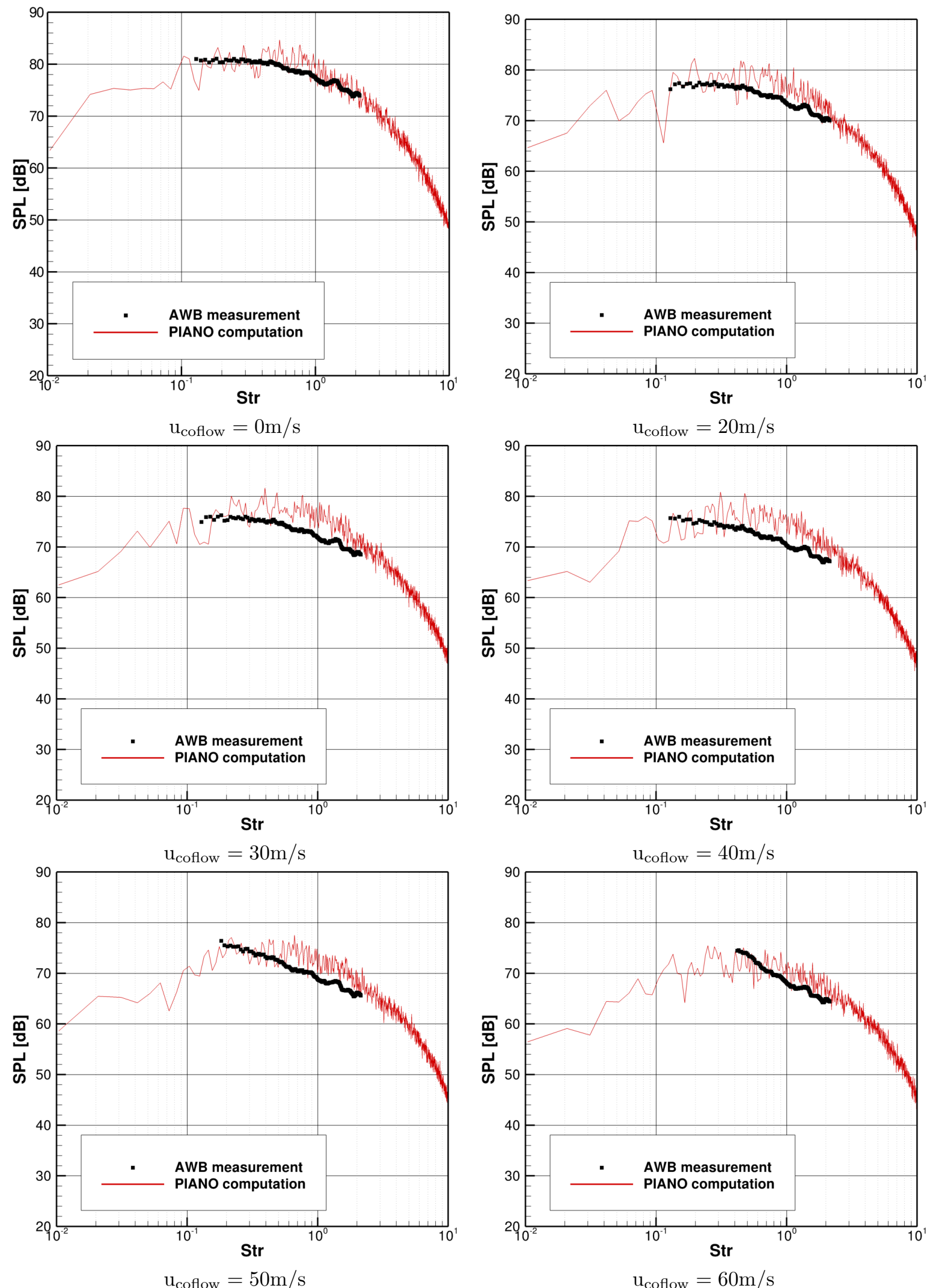

Figure 16. Computed spectra compared with AWB measurements for jet Mach number 0.6 and different wind tunnel velocities $u_{\text {coflow. }}$. All data sets are normalized to a distance of $R / D=100$. 


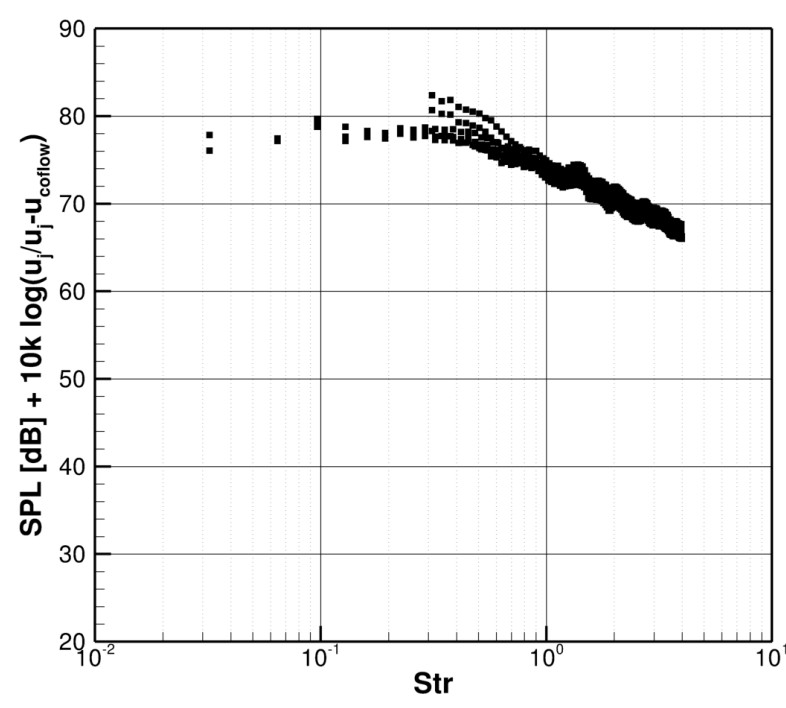

(a) AWB measurement data

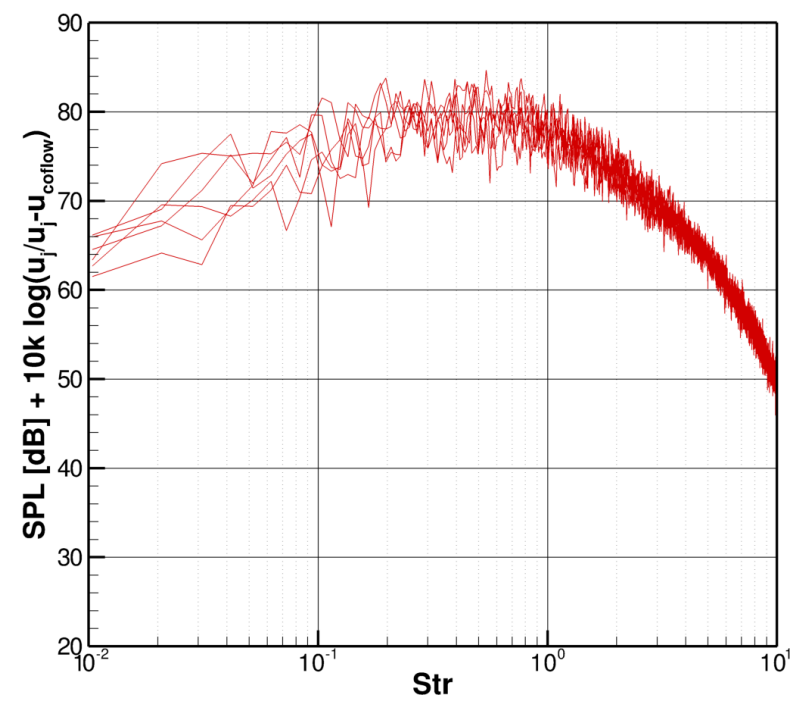

(b) PIANO simulations

Figure 17. Scaling of the normalized spectra (eg. Fig. 16) referred to Eq.(7 of measurent data (a) and computed data $(b)$

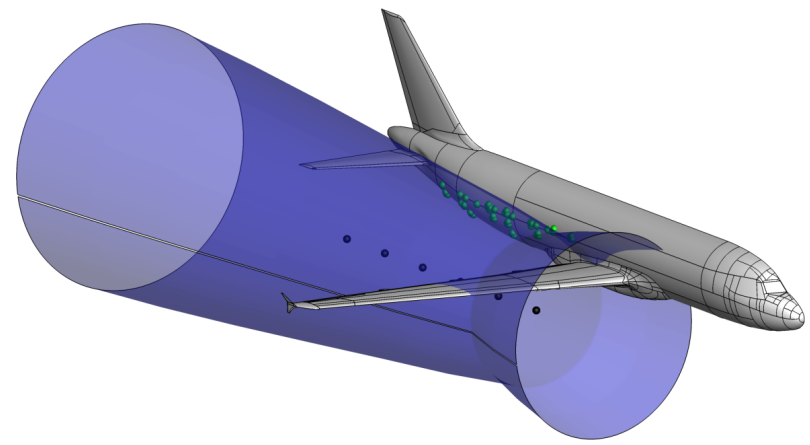

Figure 18. Extension of the revolved CAA mesh (blue surface) related to the test carrier ATRA with surface microphones positions (green dots). The black dots denotes the centerline of the CAA domain.

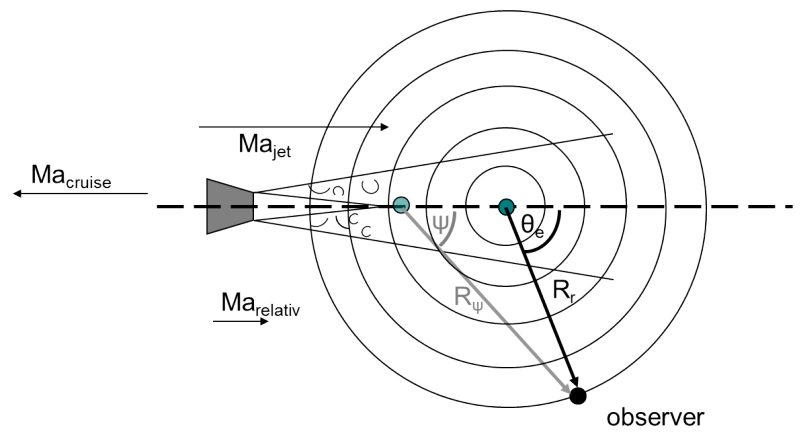

Figure 19. Jet flow at forward flight. Emission angle $\theta_{e}$ and measurement angle $\psi$ at reception time. 


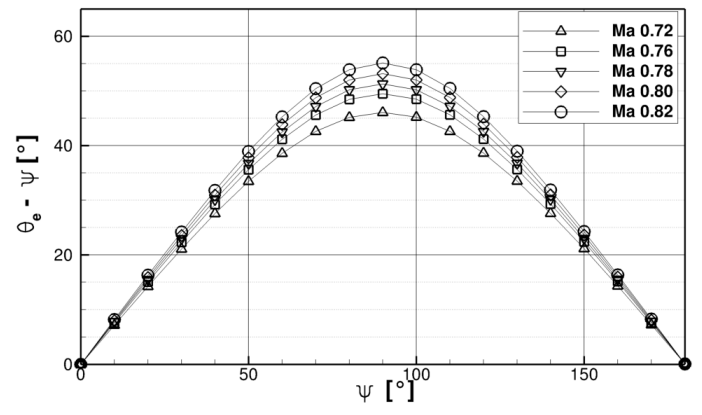

Figure 20. Difference of emission angle $\theta_{e}$ and reception angle $\psi$ at given cruise flight Mach number and reception angle

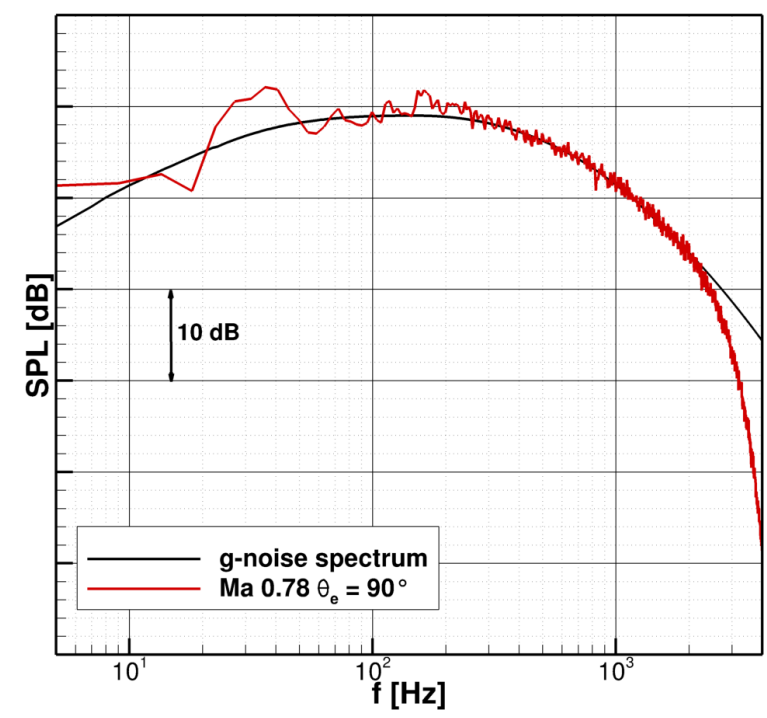

Figure 21. Comparison of generic g-noise spectrum for fine scale noise and computed spectrum at Ma 0.78 cruise flight under $90^{\circ}$ emission angle.
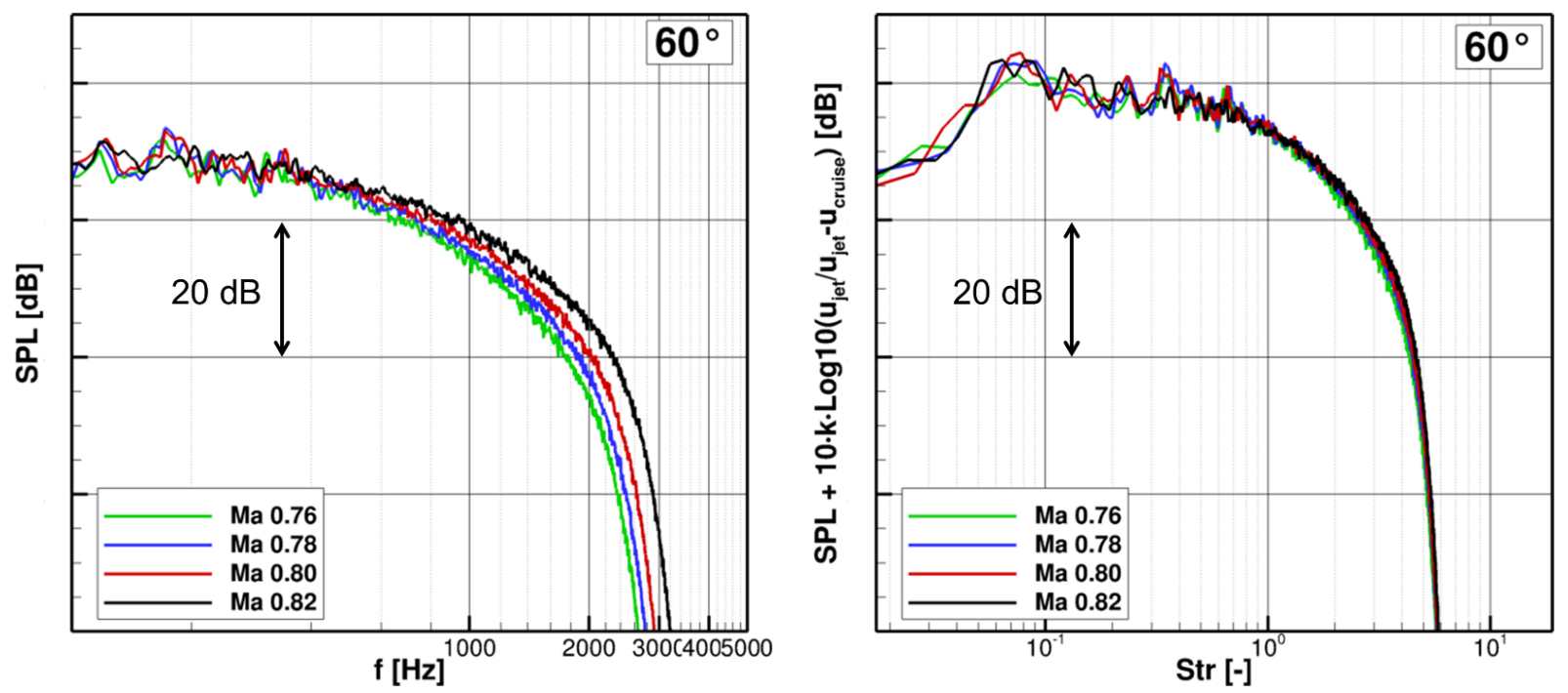

Figure 22. Effect of forward flight on jet noise spectra. Left: Narrow band spectra at serveral cruise conditions emission angle $60^{\circ}$. Right: Normalized spectra at same conditions. 

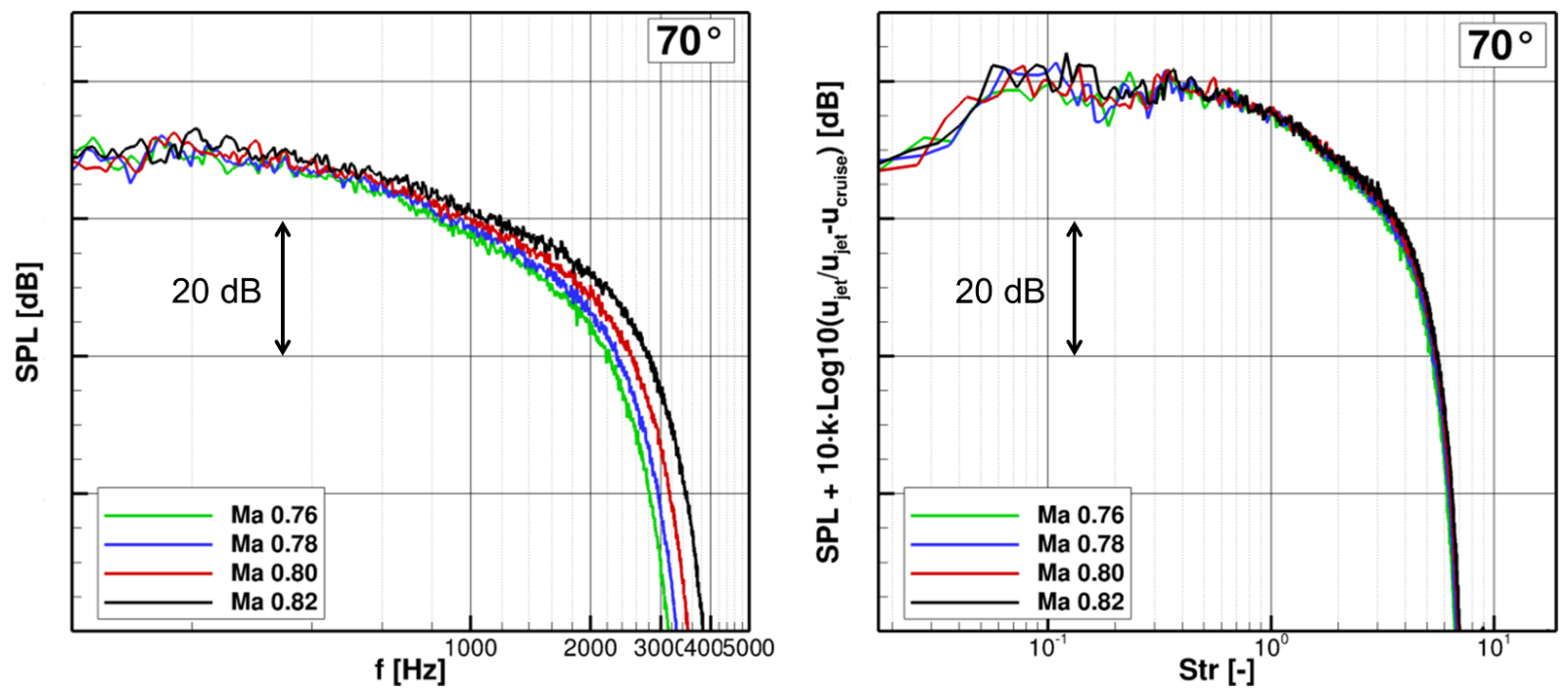

Figure 23. Effect of forward flight on jet noise spectra. Left: Narrow band spectra at serveral cruise conditions emission angle $70^{\circ}$. Right: Normalized spectra at same conditions.
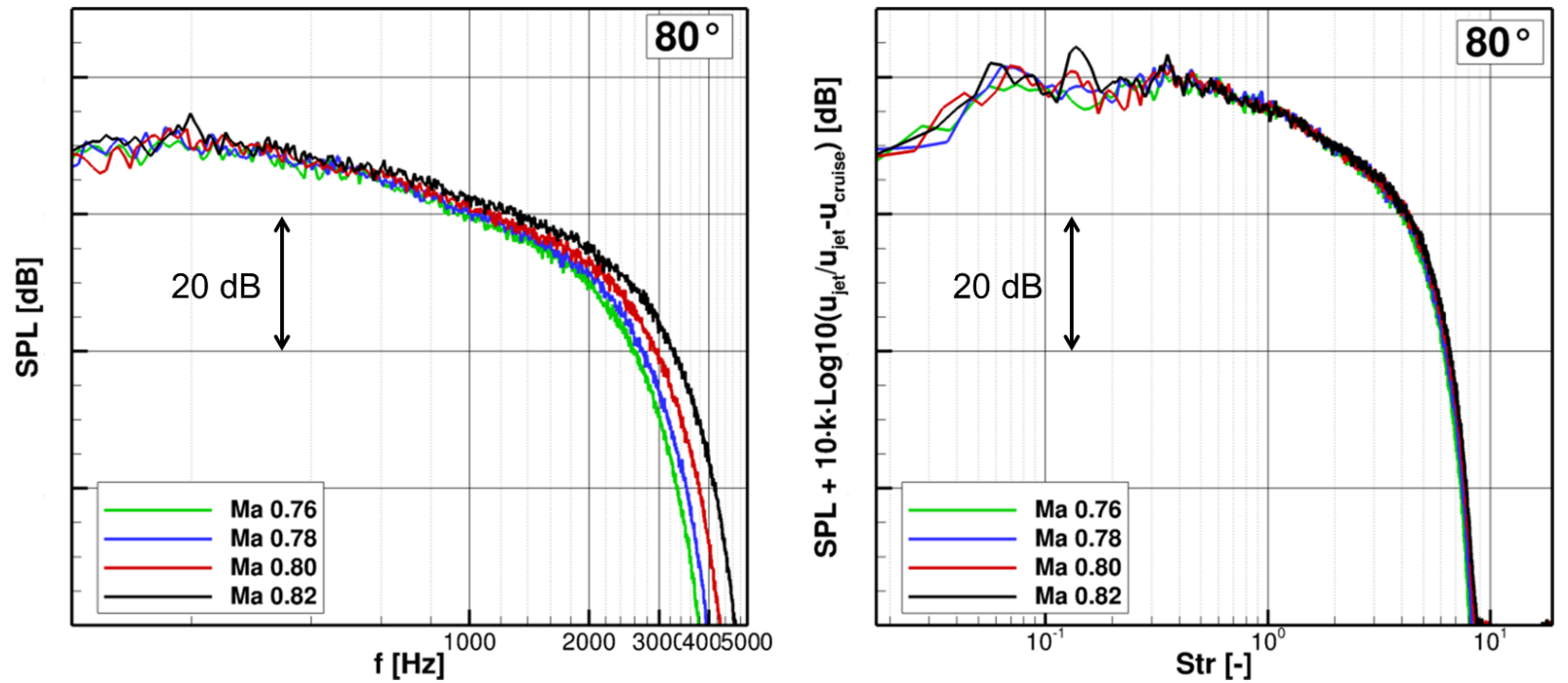

Figure 24. Effect of forward flight on jet noise spectra. Left: Narrow band spectra at serveral cruise conditions emission angle $80^{\circ}$. Right: Normalized spectra at same conditions. 

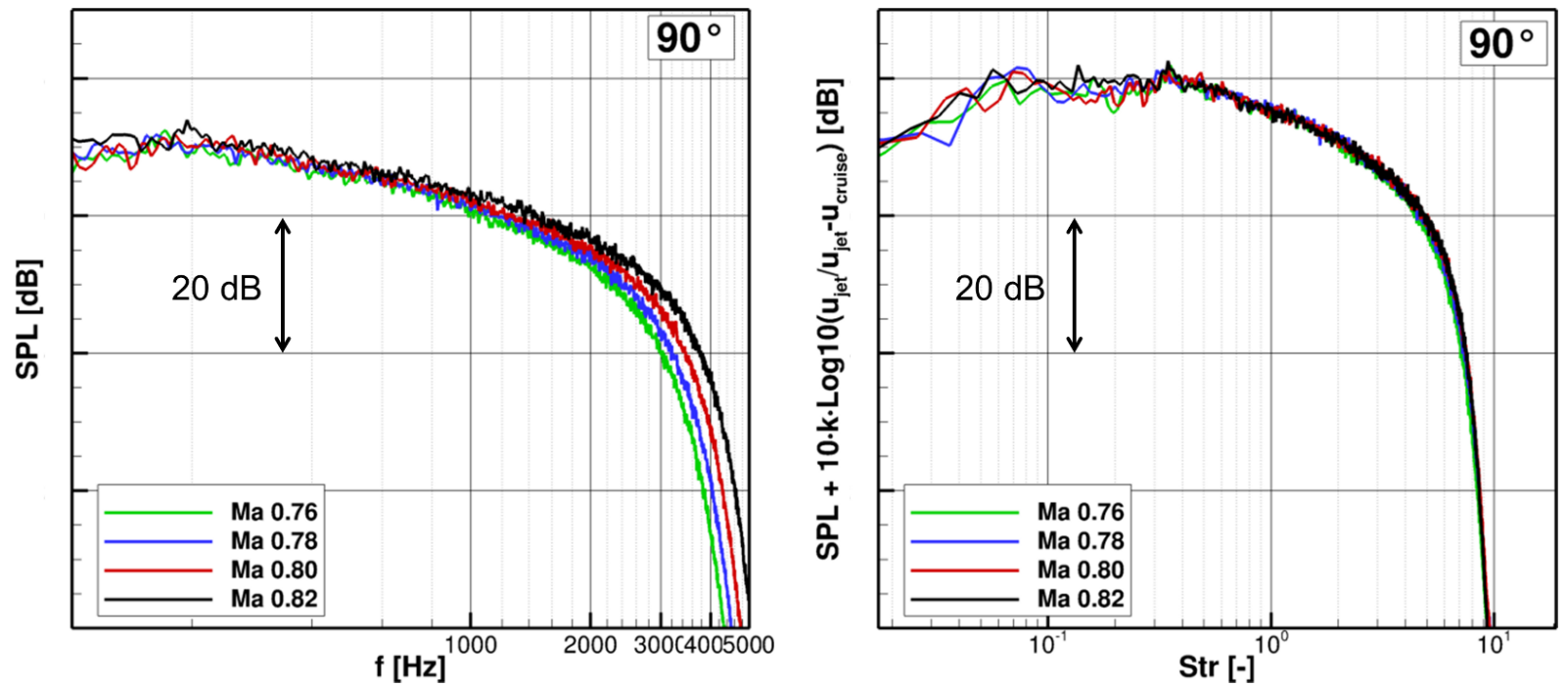

Figure 25. Effect of forward flight on jet noise spectra. Left: Narrow band spectra at serveral cruise conditions emission angle $90^{\circ}$. Right: Normalized spectra at same conditions.
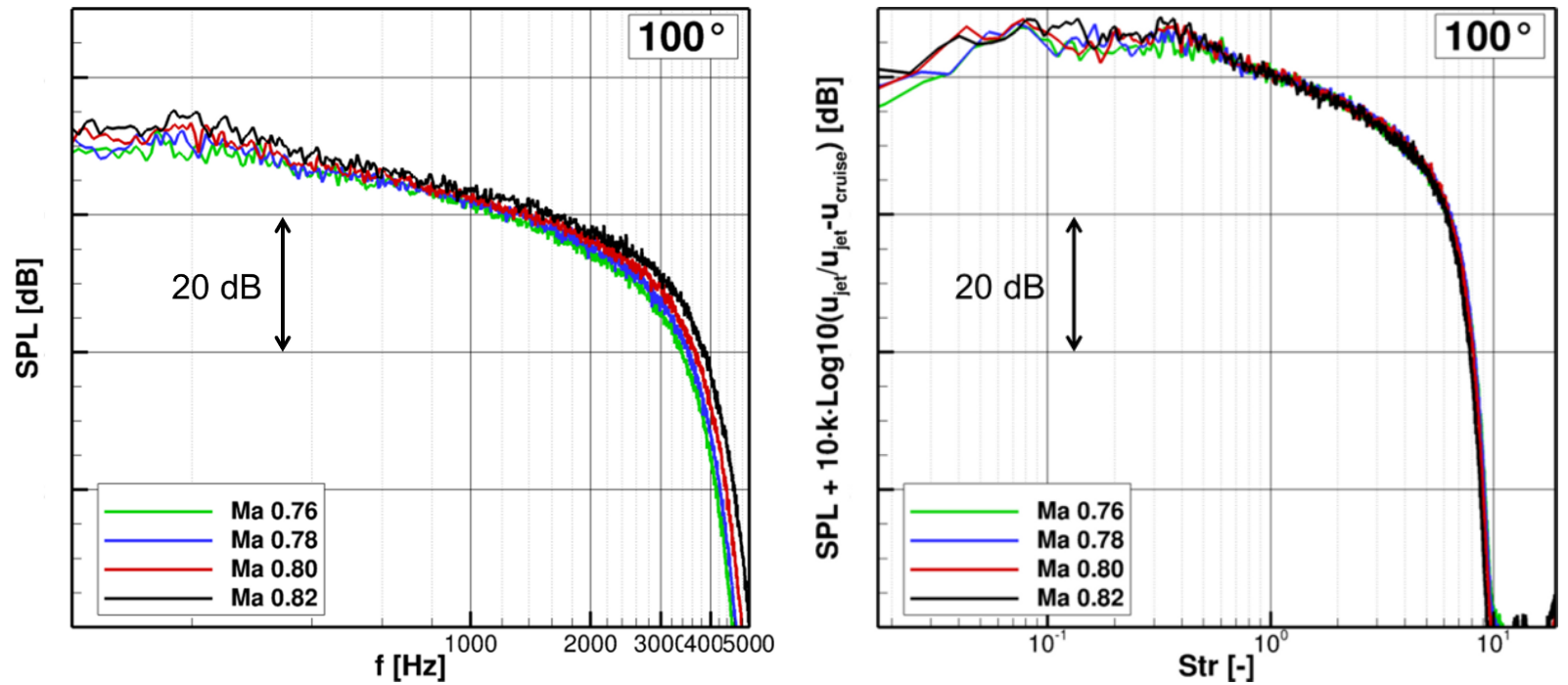

Figure 26. Effect of forward flight on jet noise spectra. Left: Narrow band spectra at serveral cruise conditions for emission angle $100^{\circ}$. Right: Normalized spectra at same conditions. 


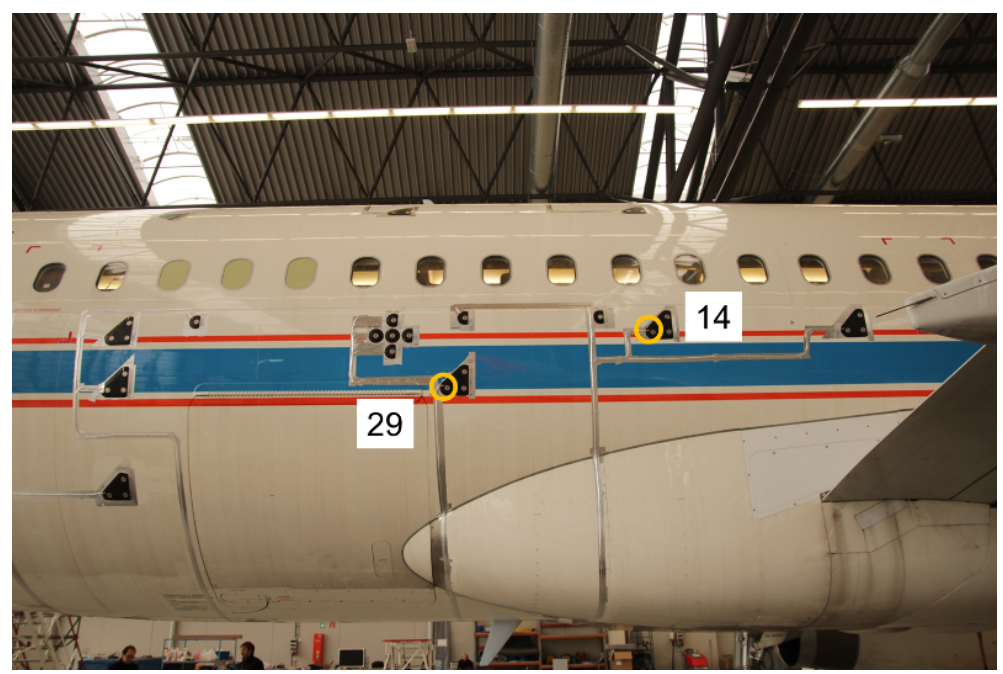

Figure 27. Surface pressure sensors at A320 ATRA fuselage

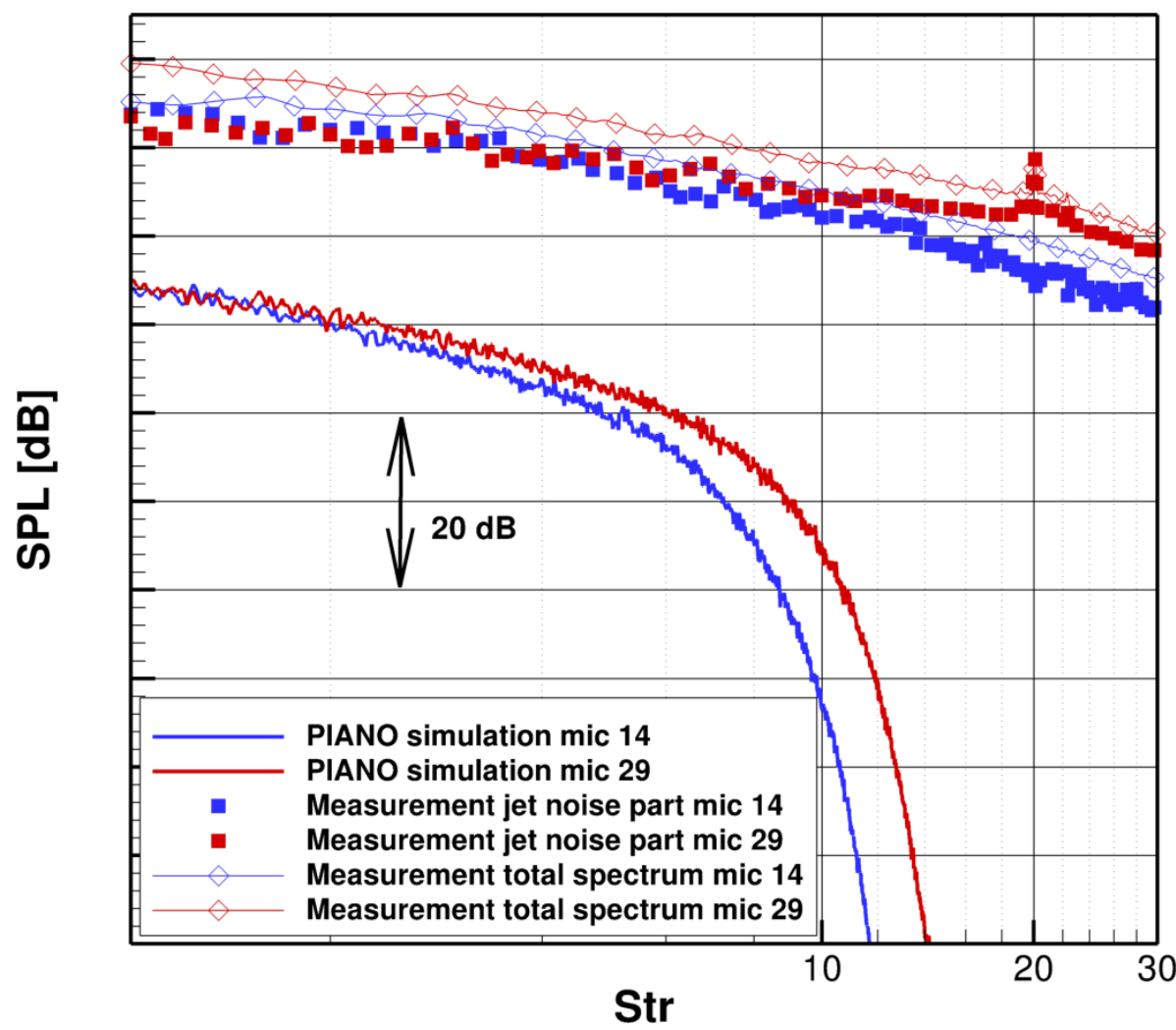

Figure 28. Computed and measured jet noise spectra at microphone positions 14 and 29 , marked in Fig. 27. Flight conditions at cruise Mach number 0.78 at FL350. Sqares mark the extracted jet noise spectra. Lines with diamond symbols show the total spectra including turbulent boundary layer excitation and jet noise. 